\title{
THE ALBANESE MAPPING FOR A PUNCTUAL HILBERT SCHEME: II. SYMMETRIZED DIFFERENTIALS AND SINGULARITIES
}

\author{
BY
}

MARK E. HUIBREGTSE

\begin{abstract}
Let $f: X \rightarrow A$ be the canonical mapping from the irreducible and nonsingular surface $X$ to its Albanese variety $A, X(n)$ the $n$-fold symmetric product of $X$, and $H_{X}^{n}$ the punctual Hilbert scheme parameterizing 0-dimensional closed subschemes of length $n$ on $X$. The latter is an irreducible and nonsingular variety of dimension $2 n$, and the "Hilbert-Chow" morphism $\sigma_{n}: H_{X}^{n} \rightarrow X(n)$ is a birational map which desingularizes $X(n)$. Let $f_{n}: X(n) \rightarrow A$ denote the map induced by $f$ by addition on $A$. This paper studies the singularities of the composite morphism

$$
\varphi_{n}: H_{X}^{n} \stackrel{\sigma_{n}}{\rightarrow} X(n) \stackrel{f_{n}}{\rightarrow} A,
$$

which is a natural generalization of the mapping $C(n) \rightarrow J$, where $C$ is an irreducible and nonsingular curve and $J$ is its Jacobian. Unlike the latter, however, $\varphi_{n}$ need not be smooth for $n \gg 0$. We prove that $\varphi_{n}$ is smooth for $n \gg 0$ only if $f: X \rightarrow A$ is smooth (Theorem 3), and over $C$ we prove the converse (Theorem 4). In case $X=A$ is an abelian surface, we show $\varphi_{n}$ is smooth for $n$ prime to the characteristic (Theorem 5), and give a counterexample to smoothness for all $n$ (Theorem 6). We exhibit a connection (over $\mathbf{C}$ ) between singularities of $\varphi_{n}$ and generalized Weierstrass points of $X$ (Theorem 9).

Our method is as follows: We first show that the singularities of $\varphi_{n}$ are the zeros of certain holomorphic 1-forms on $H_{X}^{n}$ which are the "symmetrizations" of holomorphic 1-forms on $X$. We then study "symmetrized differentials" and their zeros on $H_{X}^{n}$ (Theorems 1,2). Our method works for curves $C$ as well; we give an alternative proof of a result of Mattuck and Mayer [10, p. 226] which shows that the zeros of symmetrized differentials on $C(n)$ represent (for $C$ complete nonsingular) the special divisors of degree $n$ on $C$.
\end{abstract}

Introduction. Let $f: C \rightarrow J$ be the canonical map from the complete nonsingular curve $C$ to its Jacobian $J$. Much of the classical theory of curves can be expressed in terms of the induced map $f_{n}: C(n) \rightarrow J$, where $C(n)$ is the $n$-fold symmetric product of $C$ : The fibers of $f_{n}$ are projective spaces which represent the complete linear systems of degree $n$ on $C$; the Riemann-Roch theorem (for positive divisors) computes the dimensions of these fibers for all $n>0$. The special divisors of degree $n$ are represented on $C(n)$ by the singularities of $f_{n}$; in particular, the nonexistence of special divisors of degree $>2 g-2(g=$ genus of $C$ ) is equivalent to the smoothness of $f_{n}$ for $n>2 g-2$. The Weierstrass points of $C$ are those points $P \in C$ for

Received by the editors June 25, 1980 and, in revised form, August 11, 1981.

AMS (MOS) subject classifications (1970). Primary 14C05, 14C25, 14K99, 14J99; Secondary $14 \mathrm{H} 40$.

Key words and phrases. Punctual Hilbert scheme, symmetric product, Albanese variety, Albanese mapping, symmetrized differential, algebraic surface. 0002-9947/82/0000-0945/\$09.00 
which the divisor $g P$ is special, i.e., the point on $C(g)$ representing $g P$ is a singularity of $f_{n}$. And so on. In this paper, we study a natural higher dimensional analogue of the mapping $f_{n}$. (Note: we work over an algebraically closed ground field $k$, of arbitrary characteristic except where noted.)

Let $X$ be an irreducible and nonsingular projective variety of dimension $d>1$, and $f: X \rightarrow A$ the canonical map from $X$ to its Albanese variety $A$. The induced map $f_{n}: X(n) \rightarrow A$ is an analogue of $C(n) \rightarrow J$, but since $X(n)$ has singularities and $C(n)$ does not, the analogy is imperfect. However, when $X$ is a surface, the punctual Hilbert scheme $H_{X}^{n}$ provides a natural desingularization of $X(n)$; it is an irreducible and nonsingular complete variety of dimension $2 n$ whose points represent the closed subschemes of $X$ having Hilbert polynomial $n$. By associating to each such subscheme $Z$ the 0 -cycle $\sum_{i=1}^{r} n_{i} P_{i}$, where $\left\{P_{1}, \ldots, P_{r}\right\}=\operatorname{Supp} Z$ and $n_{i}$ is the length of $Z$ at $P_{i}$, we get a birational map

$$
\sigma_{n}: H_{X}^{n} \rightarrow X(n)
$$

(the "Hilbert-Chow" morphism), which is an isomorphism over the smooth (offdiagonal) locus of $X(n)$ [3].

We therefore study the composite morphism

$$
\varphi_{n}: H_{X}^{n} \stackrel{\sigma_{n}}{\rightarrow} X(n) \stackrel{f_{n}}{\rightarrow} A
$$

since $H_{C}^{n}=C(n)$ [8], $\varphi_{n}$ is a natural generalization of $f_{n}: C(n) \rightarrow J$. An earlier paper [6] studied the fibers of $\varphi_{n}$, and an analogue of "Riemann's theorem" was proved: all the fibers of $\varphi_{n}$ are irreducible and of dimension $2 n-q$, for $n \gg 0(q=\operatorname{dim} A)$. This paper studies the singularities of $\varphi_{n}$. Guessing from the case of curves, we began the work in an attempt to answer the following

Question. Is $\varphi_{n}$ smooth for $n \gg 0$. (And if not, where not?) We will see that the answer is, in general, no.

Our basic method for studying singularities of $\varphi_{n}$ is this: We first observe that the singularities of $\varphi_{n}$ are the zeros of certain holomorphic 1-forms on $H_{X}^{n}$, which arise by a process of "symmetrization" from holomorphic 1-forms on $X$. We therefore study "symmetrized differentials" with an eye toward finding their zeros; Theorems $1(\S 3)$ and $2(\S 4)$ are the main results in this direction. (We do not quote the theorems here since their precise statements require extensive preparations.) For curves $C$, the problem of determining the zeros of symmetrized differentials on $H_{C}^{n}=C(n)$ can be completely solved. The result $((4), \S 1)$ appears in a paper of Mattuck and Mayer [10] on the Riemann-Roch theorem for curves. Our methods work for curves as well as for surfaces, and we give an alternative proof of this result, which is the prototype of the kind of result we hoped to achieve for surfaces.

We now discuss the structure and contents of the paper in more detail. In $\$ 1$ we define symmetrized differentials and establish their connection with the singularities of $\varphi_{n} . \S \S 2$ and 3 are devoted to the formulation and proof of Theorem 1; $\S 2$ contains preliminaries on tangent bundles (especially the tangent bundle of $H_{X}^{n}$ ), and $\S 3$ contains the proof of Theorem 1 , as well as some other basic results concerning symmetrized differentials. In $\S 4$ we use the "linear determinant" [8] to prove 
Theorem 2; in tandem, Theorems 1 and 2 allow us in certain cases to find zeros of symmetrized differentials, essentially by direct computation.

In $\S 5$, we make four applications of Theorems 1 and 2 to obtain the principal results of the paper. Application 1 contains our alternative proof of (4) for curves. In Application 2, we answer the question concerning smoothness of $\varphi_{n}$ for $n \gg 0$ raised above. We prove

THEOREM 3. Let $X$ be an irreducible and nonsingular surface for which the Albanese mapping $f: X \rightarrow A$ is not smooth. Then for $n=r(r+1) / 2, r>0$, the mapping $\varphi_{n}$ : $H_{X}^{n} \rightarrow A$ is not smooth.

Over the complex numbers, we have the converse as well:

THEOREM 4. Let $X$ be an irreducible and nonsingular surface $/ \mathbf{C}$, with $q=\operatorname{dim} A>0$. Then the following are equivalent:

(i) $\varphi_{n}: H_{X}^{n} \rightarrow A$ is smooth for $n \gg 0$;

(ii) the Albanese mapping $\varphi_{1}=f: X \rightarrow A$ is smooth;

(iii) every nonzero holomorphic 1-form $\omega=f^{*}\left(\omega_{A}\right)$ has no zeros on $X\left(0 \neq \omega_{A} \in\right.$ $\left.\Gamma\left(\Omega_{A}^{1}\right)\right)$

(iv) $\varphi_{n}: H_{X}^{n} \rightarrow A$ is smooth for all $n>0$.

In particular, the conditions of Theorem 4 are satisfied when $X=A$ is an abelian surface/C. We study abelian surfaces in arbitrary characteristic in Application 3; we show that $\varphi_{n}: H_{X}^{n} \rightarrow A$ is smooth if $n$ is prime to the characteristic (Theorem 5), but that whenever $n=m^{2} \equiv 0(\bmod \operatorname{char} k), \varphi_{n}$ is not smooth (Theorem 6); indeed, it has singularities in every fiber.

In Application 4, we formulate and prove a generalization of (4) for curves to the case of surfaces (Theorem 8). This is then used in (the final) $\S 6$ to relate the differential behavior of $\varphi_{n}$ to generalized Weierstrass points on $X$ (Theorem 9), in analogy with the classical result for curves mentioned above.

I wish to thank Steven Kleiman and Arthur Mattuck for all their help, and Robert Lax, who suggested the connection between generalized Weierstrass points and singularities of $\varphi_{n}$ and inspired the results of Application 4. I also thank the referee.

Conventions. In addition to those established above, we make the following conventions. (Recall that we work over an algebraically closed ground field $k$, of arbitrary characteristic except where noted.) By a $k$-scheme we mean a separated scheme of finite type over $k$; a variety is a reduced $k$-scheme. A point of a $k$-scheme is a $k$-rational (or closed) point, unless otherwise stated. If $\sum_{i=1}^{r} n_{i} P_{i}$ is a 0 -cycle on a variety $Y$, the associated point of $Y(n)$ is written $\left(\sum_{i=1}^{r} n_{i} P_{i}\right)$, and if $Z$ is a 0-dimensional closed subscheme of length $n$ on $Y$, the associated point of $H_{Y}^{n}$ is written [ $Z$ ]. We use $C$ for a curve, $X$ for a surface, and $V$ for either, in discussions that apply to both cases. These will always be irreducible and nonsingular, but projective only when noted.

1. Symmetrized differentials and singularities of $\varphi_{n}$. Let $V$ be an irreducible and nonsingular curve or surface, $\omega$ a holomorphic l-form on $V$, and $\pi_{i}: V^{n} \rightarrow V$ the $i$ th projection map. The 1 -form $\omega^{n}=\sum_{i=1}^{n} \pi_{i}^{*}(\omega)$ on $V^{n}$ is invariant under the action of 
the symmetric group, so it induces on $V(n)$ a rational 1 -form $\omega_{(n)}$; using the morphism $\sigma_{n}: H_{V}^{n} \rightarrow V(n)$, we pull back $\omega_{(n)}$ to obtain the 1-form $\omega_{[n]}$ (the symmetrization of $\omega)$ on $H_{V}^{n}$. A priori we know that $\omega_{(n)}$ is holomorphic on the locus $V(n)_{0}=\left\{\left(P_{1}+\cdots+P_{n}\right) \mid P_{i} \neq P_{j}\right.$ for $\left.i \neq j\right\}$, over which $V^{n} \rightarrow V(n)$ is etale; thus $\omega_{[n]}$ is holomorphic on $\sigma_{n}^{-1}\left(V(n)_{0}\right)$, since $\sigma_{n}$ is an isomorphism over $V(n)_{0}$. (Of course, when $V$ is a curve, $H_{V}^{n}=V(n)$ and $\omega_{[n]}=\omega_{(n)}$.) We will show that $\omega_{[n]}$ is holomorphic everywhere on $H_{V}^{n}$ (Proposition 3); in characteristic 0 , this is a special case of [14, Theorem 3 or 11, p. 197]. It will follow that the map $\omega \mapsto \omega_{[n]}$ defines an injection $\Gamma\left(\Omega_{V}^{1}\right) \rightarrow \Gamma\left(\Omega_{H_{V}^{n}}^{1}\right)$ which is an isomorphism when $V$ is projective (Proposition 4).

We now explain the connection betwen symmetrized differentials and singularities of the Albanese mapping $\varphi_{n}: H_{V}^{n} \rightarrow A$. We first recall the following criterion for smoothness at a point:

(1) A morphism $g: S \rightarrow W$ of nonsingular varieties is smooth at $s \in S$ if and only if the differential map $D g: T S_{s} \rightarrow T W_{w}$ is surjective, where $w=g(s)$ and $T S_{s}$ and $T W_{w}$ are the tangent spaces to $S$ at $s$ and $W$ at $w$, respectively [1, VII, 5.5].

This criterion may be applied to $\varphi_{n}$ since its domain and range are nonsingular varieties. Let $[Z] \in H_{V}^{n}=H$ and $a=\varphi_{n}([Z]) \in A$. Dualized, (1) says that $\varphi_{n}$ is smooth at $[Z]$ if and only if the map $\left(D \varphi_{n}\right)^{*}: T A_{a}^{*} \rightarrow T H_{[Z]}^{*}$ of cotangent spaces is injective. But a nonzero covector at $a$ determines (and is determined by) a nonzero global holomorphic 1-form on $A$ by translation; if the pullback of this 1-form to $H$ has a zero at $[Z]$, it follows that $\left(D \varphi_{n}\right)^{*}$ is not injective. We conclude:

(2) $[Z] \in H_{V}^{n}$ is a singularity of $\varphi_{n}$ if and only if for some nonzero global holomorphic 1-form $\omega_{A}$ on $A$, the pullback $\varphi_{n}^{*}\left(\omega_{A}\right)$ is zero at [ $\left.Z\right]$.

Therefore, to determine the singularities of $\varphi_{n}$ (or prove that none exist) we have to determine the zeros of the differentials $\varphi_{n}^{*}\left(\omega_{A}\right)$ (or prove that none exist). The first (short) step is to observe that $\varphi_{n}^{*}\left(\omega_{A}\right)$ is a symmetrized differential; in fact,

(3) $\varphi_{n}^{*}\left(\omega_{A}\right)=\omega_{[n]}$, where $\omega=f^{*}\left(\omega_{A}\right)$ is the pullback of $\omega_{A}$ to $V$ by the Albanese mapping $f: V \rightarrow A$.

Proof. It is enough to prove that $f_{n}^{*}\left(\omega_{A}\right)=\omega_{(n)}$ on $V(n)$, or equivalently (separability of $\left.V^{n} \rightarrow V(n)\right)$, that the pullback of $\omega_{A}$ by the composite mapping $V^{n} \rightarrow$ $V(n) \stackrel{f_{n}}{\rightarrow} A$ is $\omega^{n}=\sum_{i=1}^{n} \pi_{i}^{*}(\omega)$. But the latter map is the sum of maps $\left(\sum_{i=1}^{n} f \circ \pi_{i}\right)$ : $V^{n} \rightarrow A$, and since the pullback of $\omega_{A}$ by a sum of maps is the sum of the pullbacks [16, Proposition 17, p. 56], we have that

$$
\left(\sum_{i=1}^{n} f \circ \pi_{i}\right)^{*}\left(\omega_{A}\right)=\sum_{i=1}^{n} \pi_{i}^{*}\left(f^{*}\left(\omega_{A}\right)\right)=\omega^{n}
$$

as desired.

REMARK. In general, the Albanese mapping $f: V \rightarrow A$ induces an injection $f^{*}$ : $\Gamma\left(\Omega_{A}^{1}\right) \rightarrow \Gamma\left(\Omega_{V}^{1}\right)$ [17]. If $V$ is complete and the base field is $\mathbf{C}$, then $f^{*}$ is an isomorphism [18, §9].

The second (long) step is to determine the zeros of symmetrized differentials on $H_{V}^{n}$. Efforts to solve this problem have led to Theorems $1(\S 3)$ and $2(\S 4)$, which 
solve the problem "in principle" if not always in practice. Briefly, these theorems enable us to compute the covector $\omega_{[n]}: T H_{[Z]} \rightarrow k$ induced at $[Z] \in H_{V}^{n}$ by the symmetrized differential $\omega_{[n]}$; if this covector is the zero map, the 1-form $\omega_{[n]}$ has a zero at $[Z]$. In practice, the computations can be carried out whenever a convenient basis of $T H_{[Z]}$ can be written down.

For example, when $V$ is a curve $C$, the problem of determining the zeros of symmetrized differentials can be completely solved:

(4) $\omega_{(n)}$ is zero at $\left(\sum_{i=1}^{r} n_{i} P_{i}\right) \in C(n) \Leftrightarrow(\omega)>\sum_{i=1}^{r} n_{i} P_{i}$, where $(\omega)$ is the divisor of $\omega$ on $C$.

This is proved in [10, p. 226] using the theory of symmetric polynomials (Newton's identities); it is a cornerstone of the proof of the Riemann-Roch theorem for curves given in the same paper. Theorems 1 and 2 can be applied in case $V=C$ to give an alternative proof of this result (Application 1, §5).

In case $V$ is a surface, no such complete solution is obtained; we have several partial results (Applications 2-4 of \$5) which yield some information about the singularities of $\varphi_{n}$, as indicated in the Introduction. Our first goal is the formulation and proof of Theorem 1 , which occupies $\S 3$; the necessary preliminaries on tangent bundles are the subject of the next section.

2. Preliminaries on tangent bundles. Let $Y$ be a $k$-scheme and let $k[\varepsilon]$ be the dual numbers $\left(\varepsilon^{2}=0\right)$. If $S$ is any $k$-scheme, we write $S[\varepsilon]$ for $S \otimes_{k} k[\varepsilon]$. Consider the contravariant functor $Y_{\varepsilon}$ from $k$-schemes to sets, whose value on the $k$-scheme $S$ is

$$
Y_{\varepsilon}(S)=\operatorname{Hom}(S[\varepsilon], Y),
$$

and whose value on the morphism $j: S^{\prime} \rightarrow S$ is the map

$$
Y_{\varepsilon}(j): \operatorname{Hom}(S[\varepsilon], Y) \rightarrow \operatorname{Hom}\left(S^{\prime}[\varepsilon], Y\right),
$$

given by composition with $j \otimes 1: S^{\prime}[\varepsilon] \rightarrow S[\varepsilon]$. If $S=\operatorname{Spec} k$, then $Y_{\varepsilon}(S)$ is the set of tangent vectors on $Y$.

(5) The functor $Y_{\varepsilon}$ is representable, and is represented by a $k$-scheme $T Y$ (the tangent bundle of $Y$ ), which is the vector bundle $V\left(\Omega_{Y / k}^{1}\right)$ associated to the sheaf of (Kähler) differentials on $Y$.

$T Y$ is defined and studied in [4, IV, 16.5.12-13] (also [2, pp. 65-72]), though it is not explicitly pointed out there that $T Y$ represents $Y_{\varepsilon}$. To see this, we must construct a natural bijection

$$
\operatorname{Hom}(S[\varepsilon], Y)=\operatorname{Hom}(S, T Y)
$$

for any $k$-scheme $S$; by standard arguments, it suffices to do this when $Y=\operatorname{Spec} B$ is affine and when $Y_{\varepsilon}$ is restricted to the category of affine $k$-schemes. In this case, by definition, $T Y=V\left(\Omega_{Y / k}^{1}\right)=\operatorname{Spec}\left(\operatorname{Symm}_{B}\left(\Omega_{B / k}^{1}\right)\right)$, where $\Omega_{B / k}^{1}$ is the module of Kahler differentials of $B$, trivial on $k$, and $\operatorname{Symm}_{B}(\cdot)$ is the functor assigning to any $B$-module its symmetric algebra.

If $S=\operatorname{Spec} R$, then a map $g: S \rightarrow T Y$ has comorphism $g^{*}: \operatorname{Symm}_{B}\left(\Omega_{B / k}^{1}\right) \rightarrow R$; in particular, $R$ becomes a $B$-algebra by the map $g_{0}^{*}: B \rightarrow R$, which is the composition of $g^{*}$ and the natural map $B \rightarrow \operatorname{Symm}_{B}\left(\Omega_{B / k}^{1}\right)$ (the comorphism of the projection map $\rho: T Y \rightarrow Y$ ). By the universal property of the symmetric algebra, $g^{*}$ 
corresponds uniquely to a $B$-module homomorphism $g^{*}: \Omega_{B / k}^{1} \rightarrow R$, which in turn corresponds uniquely to a derivation $D_{g^{*}}: B \rightarrow R$, trivial on $k$, by the universal property of $\Omega_{B / k}^{1}$. Using this derivation, one constructs a homomorphism of $k$-algebras

$$
B \rightarrow R \otimes_{k} k[\varepsilon]=R \oplus \varepsilon R, \quad b \mapsto g_{0}^{*}(b)+\varepsilon D_{g^{*}}(b),
$$

which is the comorphism of a map $g^{\prime}: S[\varepsilon] \rightarrow Y$; one checks easily that $g \mapsto g^{\prime}$ is a natural bijection, which gives (6) and establishes (5).

With $g, g^{\prime}$ as above, one sees easily that the following diagram commutes:

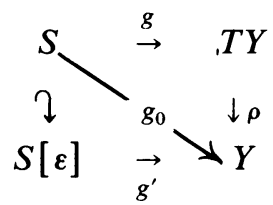

In particular, if $S=\operatorname{Spec} k$ and we fix $g_{0}$ to be the inclusion of the point $y \in Y$, we see that the fiber $\rho^{-1}(y)$ is (as expected) the tangent space $T Y_{y}$.

If $Y=\operatorname{Spec} B$ and $\Omega_{B / k}^{1}$ is free with basis $\omega_{1}, \ldots, \omega_{r}$, then $\operatorname{Symm}_{B}\left(\Omega_{B / k}^{1}\right)$ is the polynomial ring $B\left[\omega_{1}, \ldots, \omega_{r}\right]=B \otimes_{k} k\left[\omega_{1}, \ldots, \omega_{r}\right]$. Thus $T Y=\operatorname{Spec}\left(B\left[\omega_{1}, \ldots, \omega_{r}\right]\right)$ is the product of $Y$ and a vector space, linear coordinates for which are given by the $\omega_{i}$. Since the sheaf of holomorphic 1 -forms on a nonsingular variety is locally free, the tangent bundle of a nonsingular variety is locally the product of the variety and a vector space. Moreover,

(8) to give a holomorphic 1-form on a nonsingular variety $Y$ is the same as to give a holomorphic function on $T Y$ which is linear on each fiber $T Y_{y}$.

Proof. A holomorphic 1-form on $Y$ determines a linear functional (covector) on $T Y_{y}$ for all $y \in Y$; the union (roughly speaking) of these functionals gives the desired function. More precisely, it is enough to consider the case where $Y=\operatorname{Spec} B$ and $\Omega_{B / k}^{1}$ is free over $B$ with basis $\omega_{1}, \ldots, \omega_{r}$, so that $T Y=\operatorname{Spec} B\left[\omega_{1}, \ldots, \omega_{r}\right]$. Since the $\omega_{i}$ give linear coordinates on each fiber $T Y_{y}$, the holomorphic functions linear on each fiber are clearly given by the linear polynomials $\sum_{i=1}^{r} b_{i} \omega_{i} \in B\left[\omega_{1}, \ldots, \omega_{r}\right]$, but these are also exactly the elements of $\Omega_{B / k}^{1}$, and (8) follows.

We now consider the case $Y=H_{V}^{n}=H$, where $V$ is an irreducible and nonsingular curve or surface. (Actually, $V$ could be any quasiprojective $k$-scheme.) By using (5) and the universal property of $H$, the functor of points of $T H$ can be precisely described.

Let $S$ be a $k$-scheme and $g: S \rightarrow T H$ a morphism. We have a commutative diagram (as in (7))

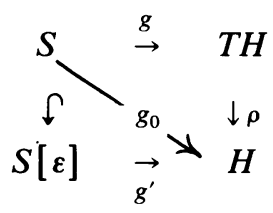

where $g$ and $g^{\prime}$ are associated by (6). For fixed $g_{0}$, we write $L_{g_{0}}$ for the set of all liftings $g$ (or $g^{\prime}$ ) making the diagram commute. By the universal property of $H$, the 
map $g_{0}$ corresponds to a closed subscheme $W_{g_{0}} \subseteq V \times_{k} S=V_{S}$, flat and finite of degree $n$ over $S$. Any $g^{\prime} \in L_{g_{0}}$ corresponds to a first order (embedded) deformation of $W_{g_{0}}$, i.e., a closed subscheme $W_{g^{\prime}} \subseteq V \times_{k} S[\varepsilon]$ that is flat over $S[\varepsilon]$ and satisfies $W_{g^{\prime}} \times_{S[\varepsilon]} S=W_{g_{0}}$. We now recall:

(9) The set of first order deformations $W_{g^{\prime}}$ of $W_{g_{0}}$ is in natural bijective correspondence with the module

$$
\Gamma\left(\underline{\text { Hom }}_{o_{v_{S}}}\left(g_{g_{0}}, O_{v_{S}} / g_{g_{0}}\right)\right),
$$

where $\mathscr{G}_{g_{0}}$ is the sheaf of ideals defining $W_{g_{0}}[5, \mathrm{IV}$, Proposition 5.1].

In case $S=\operatorname{Spec} k$, a map $g_{0}: S \rightarrow H$ corresponds to a 0 -dimensional closed subscheme $Z$ of $V$ having length $n$, and $g_{0}$ is the inclusion of the point [Z]. Then $L_{g_{0}}$ is the set of $k[\varepsilon]$-valued points (tangent vectors) of $H$ localized at [ $Z$ ], so (9) shows

$$
T H_{[Z]} \cong \Gamma\left(\underline{\operatorname{Hom}}_{o_{V}}\left(\mathcal{G}, O_{V} / \mathcal{G}\right)\right) \cong \prod_{i=1}^{r} \operatorname{Hom}_{O_{V, P_{i}}}\left(\mathscr{G}_{P_{i}}, O_{V, P_{i}} / \mathcal{G}_{P_{i}}\right),
$$

where $\mathscr{G}$ is the sheaf of ideals defining $Z$, and $\operatorname{Supp}(Z)=\left\{P_{1}, \ldots, P_{r}\right\}$ [3, p. 517].

In particular, the tangent in space $T H_{[Z]}$ is a $\Gamma\left(V, O_{V}\right)$ module, hence any $h \in \Gamma\left(V, O_{V}\right)$ defines a linear map on $T H_{[Z]}$ by multiplication. We now show that for a fixed $h$, the linear maps induced on the $T H_{[Z]}$ for various [ $\left.Z\right]$ glue together in a natural way. Recall that a bundle map $T H \rightarrow T H$ is a morphism that commutes with the projection to $H$ and is linear on each fiber $T H_{[Z]}$.

Proposition 1. Let $V, H$, and $h$ be as above. Then there exists a bundle map $\hat{h}$ : $T H \rightarrow T H$ such that for all $[Z] \in H$, the restriction of $\hat{h}$ to $T H_{[Z]}$ is the linear map given (according to (10)) by multiplication by $h$.

REMARK. In this proposition we implicitly assume that $V$ is affine, since if $V$ is projective, the holomorphic functions on $V$ are constants and the conciusion is trivial.

Proof. By (5), $T H$ represents the functor $H_{\varepsilon}$, so we may construct $\hat{h}$ as a morphism of functors.

Given a $k$-scheme $S$, we define a map

$$
\hat{h}(S): \operatorname{Hom}(S[\varepsilon], H) \rightarrow \operatorname{Hom}(S[\varepsilon], H)
$$

as follows: First break up $\operatorname{Hom}(S[\varepsilon], H)$ into the union of sets $L_{g_{0}}$ as $g_{0}$ ranges over $\operatorname{Hom}(S, H)$. Now (9) shows that $L_{g_{0}}$ is in natural bijective correspondence with the $\Gamma\left(V, O_{V}\right)$-module $\Gamma\left(\operatorname{Hom}_{O_{V}}\left(g_{g_{0}}, O_{V_{S}} / g_{g_{0}}\right)\right)$, so that multiplication by $h$ on $L_{g_{0}}$ is defined and gives a map $L_{g_{0}} \rightarrow L_{g_{0}}$; the union of these maps over all $g_{0} \in \operatorname{Hom}(S, H)$ defines $\hat{h}(S)$.

To check this gives a map of functors we must verify that for any map $j: S^{\prime} \rightarrow S$ of $k$-schemes, the induced diagram

$$
\begin{array}{ccc}
\operatorname{Hom}(S[\varepsilon], H) & \stackrel{\hat{h}(S)}{\rightarrow} & \operatorname{Hom}(S[\varepsilon], H) \\
\downarrow & & \downarrow \\
\operatorname{Hom}\left(S^{\prime}[\varepsilon], H\right) & \overrightarrow{h_{\left(S^{\prime}\right)}} & \operatorname{Hom}\left(S^{\prime}[\varepsilon], H\right)
\end{array}
$$


commutes, where the vertical arrows are defined by composition in the obvious way. This amounts to checking that for all $g_{0} \in \operatorname{Hom}(S, H)$, the diagram

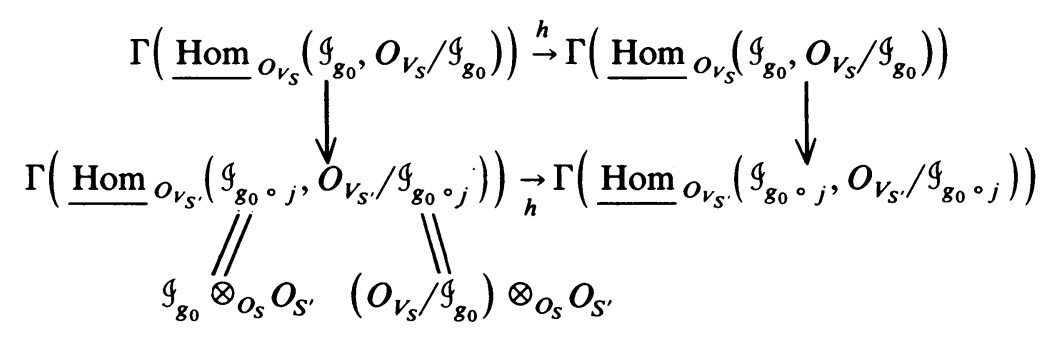

commutes, where the horizontal arrows are multiplication by $h$ and the vertical arrows are the natural ones (where $\oint_{g_{0} \circ j}$, the ideal defining $\left(W_{g_{0}}\right)_{S^{\prime}}=W_{g_{0} \circ j}$, equals $g_{g_{0}} \otimes_{O_{S}} O_{S^{\prime}}$ since $W_{g_{0}}$ is flat over $\left.O_{S}\right)$, but here commutativity is clear. Thus the bundle map $\hat{h}$ exists, and (evidently) on $T H_{[z]}, \hat{h}$ is the linear map given by multiplication by $h$ as described above, so the proposition is proved.

3. Statement of proof of Theorem 1. We begin by proving Proposition 2, which is a preliminary version of Theorem 1, and which plays a key role in the proof of Proposition 3. Let $V$ be an irreducible and nonsingular curve or surface (so that $H_{V}^{n}$ is also irreducible and nonsingular), $\omega$ a holomorphic 1-form on $V$, and $h$ a holomorphic function on $V$. We assume that $\omega_{[n]}$, the symmetrization of $\omega$, is holomorphic on $H_{V}^{n}=H$; by (8), therefore, we may view $\omega_{[n]}$ as a holomorphic function $\omega_{[n]}: T H \rightarrow k$ which is linear on each fiber. Then the composite morphism

$$
T H \stackrel{\hat{h}}{\rightarrow} T H \stackrel{\omega_{[n]}}{\rightarrow} k,
$$

where $\hat{h}$ is the bundle map given by Proposition 1 , is also a holomorphic function linear on each fiber, and so defines another holomorphic 1-form on $H$, which we write $\omega_{[n]} \circ \hat{h}$.

Proposition 2. With $V, h$, and $\omega$ as above, we have that

$$
(h \omega)_{[n]}=\omega_{[n]} \circ \hat{h}
$$

in particular; $(h \omega)_{[n]}$ is holomorphic on $H_{V}^{n}$.

For the proof of Proposition 2 and elsewhere we need the following

Remarks. Let $n_{1}, \ldots, n_{r}$ be positive integers whose sum is $n$, and let $H_{V}^{n_{i}}=H^{n_{i}}$, $H_{V}^{n}=H^{n}$. Then there is a rational map

$$
\lambda=\lambda_{n_{1}, \ldots, n_{r}}: \prod_{i=1}^{r} H^{n_{i}} \rightarrow H^{n}
$$

which is defined on the open set $U$ of points $\left(\left[Z_{1}\right], \ldots,\left[Z_{r}\right]\right)$ which satisfy Supp $Z_{i} \cap$ $\operatorname{Supp} Z_{j}=\varnothing$ for $i \neq j$, and whose value at such a point is

$$
\lambda\left(\left[Z_{1}\right], \ldots,\left[Z_{r}\right]\right)=\left[\coprod_{i=1}^{r} Z_{i}\right] \in H^{n} .
$$


The map $\lambda$ exists since the family of closed subschemes on $U$ whose member over $\left(\left[Z_{1}\right], \ldots,\left[Z_{r}\right]\right)$ is $\amalg_{i=1}^{r} Z_{i}$ is easily seen to be flat and proper.

Now let $Z$ be a closed subscheme of length $n$ with support $\left\{P_{1}, \ldots, P_{r}\right\}$, and having length $n_{i}$ at $P_{i}$. Then $Z=\amalg_{i=1}^{r} Z_{i}$, where $Z_{i}$ is supported at $P_{i}$; thus $\lambda=\lambda_{n_{1}, \ldots, n_{r}}$ is defined at $\left(\left[Z_{1}\right], \ldots,\left[Z_{r}\right]\right) \in \Pi_{i=1}^{r} H^{n_{i}}$, and $\lambda\left(\left[Z_{1}\right], \ldots,\left[Z_{r}\right]\right)=[Z]$. Let $\mathscr{G}$ be the sheaf of ideals in $O_{V}$ defining $Z$, and $G_{i}$ the ideals in the local rings $O_{P_{i}}$ defining $Z_{i}$ for $1 \leqslant i \leqslant r$. Then it can be shown that the differential map $D \lambda$ at $\left(\left[Z_{1}\right], \ldots,\left[Z_{r}\right]\right)$ is given by the following sequence of isomorphisms (recall (10)):

$$
\begin{aligned}
T\left(\prod_{i=1}^{r} H^{n_{i}}\right)_{\left(\left[Z_{1}\right], \ldots,\left[Z_{r}\right]\right)} & \cong \bigoplus_{i=1}^{r} T H_{\left[Z_{i}\right]}^{n_{i}} \cong \bigoplus_{i=1}^{r} \operatorname{Hom}_{O_{P_{i}}}\left(g_{i}, O_{P_{i}} / \mathscr{G}_{i}\right) \\
& \cong \operatorname{Hom}_{O_{V}}\left(G, O_{V} / \mathcal{G}\right) \cong T H_{[Z]}^{n} .
\end{aligned}
$$

It follows that $\lambda$ is étale at $\left(\left[Z_{1}\right], \ldots,\left[Z_{r}\right]\right)$, since it is smooth (by (1)) and quasifinite there.

Proof of Proposition 2. Since $H_{V}^{n}=H^{n}$ is nonsingular and irreducible, it is enough to show that $(h \omega)_{[n]}$ and $\omega_{[n]} \circ \hat{h}$ are equal on a nonempty open set where both are holomorphic (for then they are equal as rational 1-forms, and since $\omega_{[n]} \circ \hat{h}$ is holomorphic everywhere, so too is $(h \omega)_{[n]}$, and the equality holds everywhere). We show $(h \omega)_{[n]}=\omega_{[n]} \circ \hat{h}$ on the open set $\sigma_{n}^{-1}\left(V(n)_{0}\right)$, where $(h \omega)_{[n]}$ is known to be holomorphic.

Fix a point $[Z] \in \sigma_{n}^{-1}\left(V(n)_{0}\right)$; then $\sigma_{n}([Z])=\left(P_{1}+\cdots+P_{n}\right)$, with $P_{i} \neq P_{j}$ for $i \neq j$. Since the quotient map $V^{n} \rightarrow V(n)$ is etale over $\left(P_{1}+\cdots+P_{n}\right)$, the rational map

$$
V^{n} \rightarrow V(n) \stackrel{\sigma_{n}^{-1}}{\rightarrow} H^{n}
$$

is étale at $\left(P_{1}, \ldots, P_{n}\right)$ and is clearly equal to the rational map $\lambda=\lambda_{1, \ldots, 1}(n$ 1's $)$ as discussed above (11), where we identify $V$ and $H_{V}^{1}$. It is therefore enough to prove that the pullbacks $\lambda^{*}\left((h \omega)_{[n]}\right)$ and $\lambda^{*}\left(\omega_{[n]} \circ \hat{h}\right)$ give the same covector at $\left(P_{1}, \ldots, P_{n}\right) \in V^{n}$.

Clearly we have that $\lambda^{*}\left((h \omega)_{[n]}\right)=(h \omega)^{n}=\sum_{i=1}^{n} h_{i} \omega_{i}$, where $h_{i}=\pi_{i}^{*}(h)$ and $\omega_{i}=\pi_{i}^{*}(\omega)$. Hence, given a tangent vector $v=\left(v_{1}, \ldots, v_{n}\right) \in T V_{\left(P_{1}, \ldots P_{n}\right)}^{n} \cong \bigoplus_{i=1}^{n} T V_{P_{i}}$, we see that

$$
\begin{aligned}
\lambda^{*}\left((h \omega)_{[n]}\right)(v) & =\sum_{i=1}^{n} h_{i}\left(P_{1}, \ldots, P_{n}\right) \omega_{i}\left(v_{1}, \ldots, v_{n}\right) \\
& =\sum_{i=1}^{n} h\left(P_{i}\right) \omega\left(v_{i}\right) .
\end{aligned}
$$

We must show that $\lambda^{*}\left(\omega_{[n]} \circ \hat{h}\right)$ has the same value on the tangent vector $v$.

Let us compute:

$$
\begin{aligned}
\lambda^{*}\left(\omega_{[n]} \circ \hat{h}\right)(v) & =\omega_{[n]} \circ \hat{h}(D \lambda(v))=\omega_{[n]}\left(D \lambda\left(\hat{h}^{\prime}(v)\right)\right) \\
& =\lambda^{*}\left(\omega_{[n]}\right)\left(\hat{h}^{\prime}(v)\right)=\omega^{n}\left(\hat{h}^{\prime}(v)\right)
\end{aligned}
$$


where $\omega^{n}=\sum_{i=1}^{n} \omega_{i}$ and $\hat{h}^{\prime}$ is the linear map on $T V_{\left(P_{1}, \ldots, P_{n}\right)}^{n}$ defined by the equation $D \lambda\left(\hat{h}^{\prime}(v)\right)=\hat{h}(D \lambda(v))$ (which exists since $D \lambda: T V_{\left(P_{1}, \ldots, P_{n}\right)}^{n} \rightarrow T H_{[Z]}^{n}$ is an isomorphism).

We now compute $\hat{h}^{\prime}$. If $G$ is the sheaf of ideals defining $Z=\amalg_{i=1}^{n} P_{i}$, then the sequence of isomorphisms (12) giving $D \lambda$ in the present case is as follows $\left(M_{P_{i}}=\right.$ maximal ideal at $P_{i}$ ):

$$
\begin{aligned}
D \lambda: T V_{\left(P_{1}, \ldots, P_{n}\right)}^{n} & \cong \bigoplus_{i=1}^{n} T V_{P_{i}} \cong \bigoplus_{i=1}^{n} \operatorname{Hom}_{O_{P_{i}}}\left(M_{P_{i}}, O_{P_{i}} / M_{P_{i}}\right) \\
& \cong \operatorname{Hom}_{O_{V}}\left(G, O_{V} / \mathcal{G} \cong T H_{[Z]}^{n} .\right.
\end{aligned}
$$

With $v=\left(v_{1}, \ldots, v_{n}\right)$ as above, note that multiplication by $h$ on $D \lambda(v) \in$ $\operatorname{Hom}_{O_{V}}\left(9, O_{V} / \mathcal{G}\right)$ corresponds to multiplication of each $v_{i} \in \operatorname{Hom}_{O_{P_{i}}}\left(M_{P_{i}}, O_{P_{i}} / M_{P_{i}}\right)$ by $h$, which is the same as multiplication of each $v_{i}$ by the value $h\left(P_{i}\right)$, since $h \equiv h\left(P_{i}\right) \bmod M_{P_{i}}$. Consequently, $\hat{h}^{\prime}$ is the linear map on $T V_{\left(P_{1}, \ldots, P_{n}\right)}^{n}$ given by

$$
v=\left(v_{1}, \ldots, v_{n}\right) \mapsto\left(h\left(P_{1}\right) v_{1}, \ldots, h\left(P_{n}\right) v_{n}\right) .
$$

Using this to complete the above calculation, we get

$$
\begin{aligned}
\lambda^{*}\left(\omega_{[n]} \circ \hat{h}\right)(v) & =\omega^{n}\left(\hat{h}^{\prime}(v)\right) \\
& =\sum_{i=1}^{n} \omega_{i}\left(h\left(P_{1}\right) v_{1}, \ldots, h\left(P_{n}\right) v_{n}\right) \\
& =\sum_{i=1}^{n} h\left(P_{i}\right) \omega\left(v_{i}\right)=\lambda^{*}\left((h \omega)_{[n]}\right)(v),
\end{aligned}
$$

which proves the proposition.

Now we drop the assumption that $\omega_{[n]}$ is holomorphic in order to prove it so:

Proposition 3. Let $V$ be an irreducible and nonsingular curve or surface, and $\omega$ a holomorphic 1-form on $V$. Then $\omega_{[n]}$ is holomorphic on $H_{V}^{n}$.

Proof. Let $[Z] \in H_{V}^{n}$, and let $U=\operatorname{Spec} R$ be an affine open subset of $V$ containing the finite set $\operatorname{Supp} Z$. ( $U$ exists since $V$ is quasiprojective-every nonsingular curve or surface is quasiprojective [19, vol II.2.6].) Then $H_{U}^{n}$ is open in $H_{V}^{n}$, and $[Z] \in H_{U}^{n}$; we complete the proof by showing that the restriction $\omega_{[n]} \mid H_{U}^{n}$ is holomorphic on $H_{U}^{n}$.

But clearly

$$
\omega_{[n]} \mid H_{U}^{n}=(\omega \mid U)_{[n]}
$$

moreover, $\omega \mid U$, being a holomorphic 1-form on $U=\operatorname{Spec} R$ (i.e., an element of $\left.\Omega_{R / k}^{1}\right)$, may be written

$$
\omega \mid U=\sum_{\alpha=1}^{r} h_{\alpha} d g_{\alpha}
$$

where $g_{\alpha}, h_{\alpha} \in R[15$, Proposition 1, p. 159]. Thus

$$
\omega_{[n]} \mid H_{U}^{n}=(\omega \mid U)_{[n]}=\left(\sum_{\alpha=1}^{r} h_{\alpha} d g_{\alpha}\right)_{[n]}=\sum_{\alpha=1}^{r}\left(h_{\alpha} d g_{\alpha}\right)_{[n]}
$$


where we have used the obvious linearity of $\omega \mapsto \omega_{[n]}$ to obtain the last equality. It suffices now to prove that each summand $\left(h_{\alpha} d g_{\alpha}\right)_{[n]}$ is holomorphic; if we knew that each $\left(d g_{\alpha}\right)_{[n]}$ were holomorphic, Proposition 2 would apply to yield the desired conclusion. It remains therefore to prove

(13) if $g$ is a holomorphic function on $U$, then the symmetrized differential $(d g)_{[n]}$ is holomorphic on $H_{U}^{n}$. (Here $U$ need not be affine.)

Proof. First note that on $U^{n}$,

$$
(d g)^{n}=\sum_{i=1}^{n} \pi_{i}^{*}(d g)=d\left(\sum_{i=1}^{n} g_{i}\right),
$$

where $g_{i}=\pi_{i}^{*}(g)$; since $\sum_{i=1}^{n} g_{i}$ is symmetric, it determines a holomorphic function $g_{(n)}$ on $V(n)$, and $(d g)_{(n)}=d\left(g_{(n)}\right)$. Thus

$$
(d g)_{[n]}=\sigma_{n}^{*}\left((d g)_{(n)}\right)=\sigma_{n}^{*}\left(d\left(g_{(n)}\right)\right)=d\left(\sigma_{n}^{*}\left(g_{(n)}\right)\right)
$$

is holomorphic, since $\sigma_{n}^{*}\left(g_{(n)}\right)$ is a holomorphic function on $H_{U}^{n}$; thus (13) holds and Proposition 3 is proved.

Propositions 2 and 3 are summarized in

THEOREM 1. Let $V$ be an irreducible and nonsingular curve or surface, $h a$ holomorphic function on $V$, and $\omega$ a holomorphic 1-form on $V$. Then the 1-forms $\omega_{[n]}$, $(h \omega)_{[n]}$, and $\omega_{[n]} \circ \hat{h}$ are holomorphic on $H_{V}^{n}$, and

$$
(h \omega)_{[n]}=\omega_{[n]} \circ \hat{h},
$$

where $\hat{h}$ is the bundle map given by Proposition 1.

Proof. Since $\omega_{[n]}$ is now known to be holomorphic (Proposition 3), Proposition 2 applies.

COROLLARY 1. If $g$ and $h$ are holomorphic functions on $V$, then

$$
(d(g h))_{[n]}=(d g)_{[n]} \circ \hat{h}+(d h)_{[n]} \circ \hat{g}
$$

(which for $n=1$ is the Leibniz rule on $V=H_{V}^{1}$ ).

Proof. Since $d(g h)=h d g+g d h$ on $V$, the corollary follows immediately from the theorem and the linearity of the map $\omega \mapsto \omega_{[n]}$.

COROLlaRY 2. If $\hat{h}$ is the zero map on $T H_{[Z]}\left(H=H_{V}^{n}\right)$, then $(h \omega)_{[n]}$ is zero at $[Z] \in H$ for all holomorphic 1-forms $\omega$ on $V$.

REMARK. The hypothesis on $\hat{h}$ holds, for example, whenever $h \in \mathcal{G}$, where $\mathcal{G}$ is the sheaf of ideals defining $Z$, since (10)

$$
T H_{[Z]}=\Gamma\left(\underline{\operatorname{Hom}}_{o_{V}}\left(q, O_{V} / q\right)\right)
$$

is killed by multiplication by any elements of $\mathscr{Y}$.

Proof. Since $(h \omega)_{[n]}=\omega_{[n]} \circ \hat{h}$ by the theorem, the corollary is obvious.

Corollary 2 suggests one way of finding zeros of symmetrized differentials, which we exploit in Proposition 6 of Application 2. More generally, Theorem 1 reduces the problem of computing the induced covector $\omega_{[n]}: T H_{[Z]} \rightarrow k$ to the case $\omega_{[n]}=d g_{[n]}$ 
is the symmetrization of an exact 1-form on $V$. Indeed, replacing $V$ by an affine open subset containing $\operatorname{Supp} Z$, we may write $\omega=\Sigma_{\alpha} h_{\alpha} d g_{\alpha}$ (as in the proof of Proposition 3), where $h_{\alpha}$ and $g_{\alpha}$ are holomorphic on $V$. Then $\omega_{[n]}=\left(\Sigma_{\alpha} h_{\alpha} d g_{\alpha}\right)_{[n]}=$ $\Sigma_{\alpha}\left(d g_{\alpha}\right)_{[n]} \circ \hat{h}_{\alpha}$, so the covector $\omega_{[n]}: T H_{[Z]} \rightarrow k$ can be computed by summing the covectors $T H_{[Z]} \stackrel{\hat{h}_{\alpha}}{\rightarrow} T H_{[Z]} \stackrel{\left(d g_{\alpha}\right)_{[n]}}{\rightarrow} k$. In the next section, we show how the covector induced by $\left(d g_{\alpha}\right)_{[n]}$ can be computed explicitly (Theorem 2). Meanwhile, for completeness, we prove

Proposition 4. Let $V$ be a nonsingular and irreducible curve or surface. Then the map $\omega \mapsto \omega_{[n]}$ defines an injection $\Gamma\left(\Omega_{V}^{1}\right) \rightarrow \Gamma\left(\Omega_{H_{V}^{n}}^{1}\right)$, which is an isomorphism if $V$ is projective.

Proof. Since $\omega_{[n]}$ is holomorphic by Proposition 3, the map is defined, is clearly linear, and is injective since

$$
\begin{aligned}
\omega_{[n]}=0 \text { on } H_{V}^{n} & \Rightarrow \omega_{(n)}=0 \text { on } V(n) \\
& \Rightarrow \omega^{n}=0 \text { on } V^{n} \\
& \Rightarrow \omega=0 \text { on } V .
\end{aligned}
$$

It remains to prove, assuming $V$ is projective, that the map is surjective.

Let $\omega_{H}$ be a holomorphic 1-form on $H_{V}^{n}$, and let $\omega_{V(n)}$ be the rational 1-form on $V(n)$ corresponding to $\omega_{H}$ by the birational map $\sigma_{n}: H_{V}^{n} \rightarrow V(n)$. Let $\omega_{V^{n}}$ be the pullback of $\omega_{V(n)}$ to $V^{n}$. In case $V$ is a curve, $V(n)=H_{V}^{n}$ and $\omega_{V(n)}=\omega_{H}$ is holomorphic on $V(n)$; hence $\omega_{V^{n}}$ is holomorphic on $V^{n}$. In case $V$ is a surface, $\omega_{V^{n}}$ is holomorphic off the branch locus of $V^{n} \rightarrow V(n)$, which has codimension $>1$ for $n>1$; hence $\omega_{V^{n}}$ is again holomorphic. In either case, $\omega_{V^{n}}$ is invariant under the action of the symmetric group, and since any (global) holomorphic 1-form on a product of projective varieties is a sum of 1 -forms coming from the factors, we see that $\omega_{V^{n}}=\sum_{i=1}^{n} \pi_{i}^{*}(\omega)$ for some holomorphic 1-form $\omega$ on $V$. Using the separability of $V^{n} \rightarrow V(n)$, we conclude that $\omega_{V(n)}=\omega_{(n)}$; it follows that $\omega_{H}=\omega_{[n]}$, which proves the surjectivity.

4. The linear determinant and Theorem 2. As above, we let $V$ be an irreducible and nonsingular curve or surface (although much of this section remains valid when $V$ is an arbitrary quasiprojective variety). The goal of this section is the proof of Theorem 2, which gives a formula for the covector induced on $T H_{[Z]}\left(H=H_{V}^{n}\right)$ by a symmetrized differential $d g_{[n]}$, where $d g$ is an exact 1 -form holomorphic on a neighborhood of Supp $Z \subseteq V$. This formula can be computed explicitly whenever a "convenient" basis of $T H_{[Z]}$ can be written down.

The proof of Theorem 2 is essentially a direct computation made possible by an explicit description (given in [8]) of the morphism $\sigma_{n}: H_{V}^{n} \rightarrow V(n)$ as a morphism of functors (of points). In the proof, we apply this description of $\sigma_{n}$ to $k[\varepsilon]$-valued points (tangent vectors) of $H_{V}^{n}$.

We now summarize what we need from [8]. Let $T$ be a $k$-scheme, and $v: T \rightarrow H_{V}^{n}$ a $T$-valued point of $H_{V}^{n}$, which corresponds to a closed subscheme $W=W_{v} \subseteq V \times T$, flat and finite of degree $n$ over $T$. In [8, II.2] there is constructed a map

$$
\theta_{W / T}: T \rightarrow W_{T}(n),
$$


called the canonical section of $W / T$, whose range is the $n$-fold symmetric product of $W$ over $T$. If $W(t)$ is the fiber of $W$ over $t \in T$, then $\theta_{W / T}(t)=\left(\sum_{i=1}^{r} n_{i} P_{i}\right)$, where Supp $W(t)=\left\{P_{1}, \ldots, P_{r}\right\}$ and $n_{i}$ is the length of $W(t)$ at $P_{i}$. From $v$, we obtain a $T$-valued point of $V(n)$ by the composite map

$$
\begin{gathered}
v_{\sigma}: T \stackrel{\theta_{W / T}}{\rightarrow} W_{T}(n) \rightarrow(V \times T)_{T}(n) \rightarrow V(n) ; \\
V(n){ }^{\|} \times_{k} T
\end{gathered}
$$

the resulting map $v \mapsto v_{\sigma}$ of $T$-valued points is functorial and corresponds to the morphism $\sigma_{n}: H_{V}^{n} \rightarrow V(n)$.

What we need to get our hands on is the comorphism of $v_{\sigma}$, in the case where $V=\operatorname{Spec} R$ and $T=\operatorname{Spec} A$ are affine. Then $W=W_{v}=\operatorname{Spec}(R \otimes A) / I_{v}$, where $(R \otimes A) / I_{v}=M_{v}=M$ is a finite and flat $A$-algebra, locally free of dimension $n$. For a $B$-module $N$, we write $N_{B}^{(n)} \subseteq \otimes_{B}^{n} N$ for the $B$-module of symmetric tensors. The comorphism of the map $v_{\sigma}(15)$ is then given by the map

$$
v_{\sigma}^{*}: R_{k}^{(n)} \rightarrow\left(R \otimes_{k} A\right)_{A}^{(n)} \rightarrow M_{A}^{(n)} \stackrel{\theta_{W / T}^{*}}{\rightarrow} A,
$$

where only the last arrow (the comorphism of the canonical section (14)) requires further explanation: it is the horizontal arrow in the commutative diagram

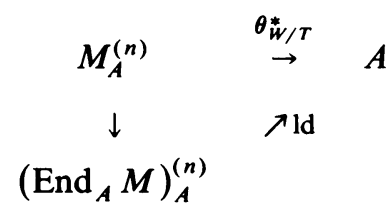

where the vertical arrow is induced by the natural map $M \rightarrow \operatorname{End}_{A} M$ (sending $m \in M$ to the $A$-linear map given by multiplication by $m$ ), and the diagonal arrow is the linear determinant (ld).

If $M$ is a free module of dimension $n$ over $A$, the linear determinant is defined as follows: let $\left(\operatorname{End}_{A} M\right)_{A}^{(n)}$ act on the 1-dimensional $A$-module $\Lambda_{A}^{n} M$ in the obvious way, so that every element $\rho \in\left(\operatorname{End}_{A} M\right)_{A}^{(n)}$ corresponds to multiplication by a scalar on $\Lambda_{A}^{n} M$. By definition, this scalar is $\operatorname{ld}(\rho)$.

For example, let $\lambda \in$ End $_{A} M$. Form the $n$-fold symmetric tensors

$$
\lambda_{(n)}=\sum_{i=1}^{n} 1 \otimes \cdots \otimes 1 \otimes \lambda \otimes 1 \otimes \cdots \otimes 1 \quad(\lambda \text { in } i \text { th place })
$$

and

$$
\lambda^{(n)}=\lambda \otimes \lambda \otimes \cdots \otimes \lambda,
$$

and choose a basis $m_{1}, \ldots, m_{n}$ for $M$ over $A$. By definition of ld, we see that

$$
\sum_{i=1}^{n} m_{1} \wedge m_{2} \wedge \cdots \wedge \lambda\left(m_{i}\right) \wedge \cdots \wedge m_{n}=\operatorname{ld}\left(\lambda_{(n)}\right) \cdot\left(m_{1} \wedge m_{2} \wedge \cdots \wedge m_{n}\right),
$$

and

$$
\lambda\left(m_{1}\right) \wedge \lambda\left(m_{2}\right) \wedge \cdots \wedge \lambda\left(m_{n}\right)=\operatorname{ld}\left(\lambda^{(n)}\right) \cdot\left(m_{1} \wedge m_{2} \wedge \cdots \wedge m_{n}\right)
$$


hence

$$
\operatorname{ld}\left(\lambda_{(n)}\right)=\operatorname{tr} \lambda \text { and } \operatorname{ld}\left(\lambda^{(n)}\right)=\operatorname{det} \lambda .
$$

Remaining in the case $V=\operatorname{Spec} R, T=\operatorname{Spec} A$, etc., let $g$ be a holomorphic function on $V(g \in R)$, and let $g_{(n)}$ (as in the proof of (13)) denote its symmetrization on $V(n)$. As a symmetric tensor, $g_{(n)}=\sum_{i=1}^{n} 1 \otimes \cdots \otimes g \otimes \cdots \otimes 1 \in R_{k}^{(n)}(g$ in $i$ th place). Viewing $g$ as an element of $R, R \otimes A$, and $R \otimes A / I_{v}=M_{v}$ simultaneously, we write $g: M_{v} \rightarrow M_{v}$ for the $A$-linear map given by multiplication by $g$. If we assume $M_{v}$ is free over $A$, then (16), (17), and (18) combine to prove

$$
v_{\sigma}^{*}\left(g_{(n)}\right)=\operatorname{tr}\left(g: M_{v} \rightarrow M_{v}\right) \in A ;
$$

this fact is the heart of the proof of Theorem 2.

We now turn to the statement of Theorem 2. Let $V=\operatorname{Spec} R, Z=\operatorname{Spec} R / I$ be a 0 -dimensional closed subscheme of length $n$ on $V$, and $g$ be a holomorphic function on $V$. The symmetrized differential $d g_{[n]}$ induces a covector on $T H_{[Z]}$, and we write $d g_{[n]}(v)$ for the value this covector has on $v \in T H_{[Z]}$. Our goal is to derive a formula for $d g_{[n]}(v)$; to this end, we express $v$ as a map $v: T=\operatorname{Spec} k[\varepsilon] \rightarrow H_{V}^{n}$ localized on [ $Z]$. As above, the map $v$ corresponds to a closed subscheme $W_{v} \subseteq V \times T$ which is flat and finite of degree $n$ over $T$; thus, $W_{v}=\operatorname{Spec}\left((R \otimes k[\varepsilon]) / I_{v}\right)$, where $(R \otimes k[\varepsilon]) / I_{v}=M_{v}$ is flat (and therefore free) of dimension $n$ over $k[\varepsilon]$, and $I_{v} \otimes_{k[\varepsilon]} k=I$. As a convenient notation, given $a+\varepsilon b \in k[\varepsilon](a, b \in k)$, we call $b$ the $\varepsilon$-coefficient of $a+\varepsilon b$, and write $C_{\varepsilon}(a+\varepsilon b)=b$. We may now state

THEOREM 2. With $V, Z, v \in T H_{[Z]}$, g, etc., as above, we have that

$$
d g_{[n]}(v)=C_{\varepsilon}\left(\operatorname{tr}\left(g: M_{v} \rightarrow M_{v}\right)\right),
$$

where $g: M_{v} \rightarrow M_{v}$ is the $k[\varepsilon]$-linear map given by multiplication by $g$ on $M_{v}$.

Before proceeding with the proof, we need the following

REMARKS. Let $U$ be a variety and $u \in U$. A tangent vector to $U$ at $u$ is usually defined to be an element $v \in \operatorname{Der}_{k}\left(O_{U, u}, k\right) \approx \operatorname{Hom}_{k}\left(M_{u} / M_{u}^{2}, k\right)$, and when so defined, the covector $d \rho$ is given by the equation

$$
d \rho(v)=v(\rho)
$$

for all $\rho \in O_{U, u}[2$, p. 65]. Furthermore, when $v$ is given as a map $v: T=\operatorname{Spec} k[\varepsilon] \rightarrow$ $U$, the derivation $v: O_{U, u} \rightarrow k$ is recovered from the comorphism $v^{*}: O_{U, u} \rightarrow k[\varepsilon]=$ $k \oplus \varepsilon k$ by the formula

$$
v(\rho)=C_{\varepsilon}\left(v^{*}(\rho)\right) .
$$

Thus, when a tangent vector is given as a map $v: T \rightarrow U$, we have that

$$
d \rho(v)=C_{\varepsilon}\left(v^{*}(\rho)\right), \quad \rho \in O_{U, u} .
$$

Proof of Theorem 2. As we saw in the proof of (13),

$$
d g_{[n]}=\sigma_{n}^{*}\left(d g_{(n)}\right)=d\left(\sigma_{n}^{*}\left(g_{(n)}\right)\right)
$$


is an exact holomorphic 1 -form on $H_{V}^{n}$. Thus

$$
\begin{aligned}
d g_{[n]}(v) & =d\left(\sigma_{n}^{*}\left(g_{(n)}\right)\right)(v) \\
& =C_{\varepsilon}\left(v^{*}\left(\sigma_{n}^{*}\left(g_{(n)}\right)\right)\right) \quad(\text { by } 20) \\
& =C_{\varepsilon}\left(v_{\sigma}^{*}\left(g_{(n)}\right)\right) \quad\left(\text { since } v_{\sigma}=\sigma_{n} \circ v\right) \\
& =C_{\varepsilon}\left(\operatorname{tr}\left(g: M_{v} \rightarrow M_{v}\right)\right) \quad(\text { by }(19)),
\end{aligned}
$$

as desired.

5. Applications of Theorems 1 and 2. We now apply Theorems 1 and 2 to obtain the results outlined in the Introduction. Before proceeding with the applications, we need to make some technical preparations. Let $V$ be an irreducible and nonsingular curve or surface, $\omega$ a holomorphic 1 -form on $V$, and $Z$ a 0 -dimensional closed subscheme of $V$ of length $n$, with support $\left\{P_{1}, \ldots, P_{r}\right\}$; thus $Z=\amalg_{i=1}^{r} Z_{i}$, where $Z_{i}$ has length $n_{i}$ and is concentrated at $P_{i}$.

Proposition 5. The 1-form $\omega_{[n]}$ is zero at $[Z] \in H_{V}^{n}=H^{n}$ if and only if each $\omega_{\left[n_{1}\right]}$ is zero at $\left[Z_{i}\right] \in H^{n_{i}}=H^{n_{i}}$, for $1 \leqslant i \leqslant r$.

Proof. By the remarks that preceded the proof of Proposition 2, there is a rational map (11)

$$
\lambda=\lambda_{n_{1}, \ldots, n_{r}}: \prod_{i=1}^{r} H^{n_{i}} \rightarrow H^{n},
$$

defined and étale at $\left(\left[Z_{1}\right], \ldots,\left[Z_{r}\right]\right)$, with $\lambda\left(\left[Z_{1}\right], \ldots,\left[Z_{r}\right]\right)=[Z]$. We claim that

$$
\lambda^{*}\left(\omega_{[n]}\right)=\sum_{i=1}^{r} p r_{i}^{*}\left(\omega_{\left[n_{i}\right]}\right),
$$

where $p r_{i}$ is the $i$ th projection; the proposition follows immediately from the claim, since $\lambda$ is étale at $\left(\left[Z_{1}\right], \ldots,\left[Z_{r}\right]\right)$.

To prove the claim, note that the diagram

$$
\begin{array}{rcc}
\prod_{i=1}^{r} H^{n_{i}} & \stackrel{\lambda}{\rightarrow} & H^{n} \\
\sigma_{n_{1}} \times \cdots \times \sigma_{n_{r}} \downarrow & & \downarrow \sigma_{n} \\
\prod_{i=1}^{r} V\left(n_{i}\right) & \rightarrow & V(n)
\end{array}
$$

commutes (on $\operatorname{dom}(\lambda)$ ), where $\alpha$ is the map that adds 0 -cycles. It is therefore enough to prove that $\alpha^{*}\left(\omega_{(n)}\right)=\sum_{i=1}^{r} p r_{i}^{*}\left(\omega_{\left(n_{i}\right)}\right)$ on $\prod_{i=1}^{r} V\left(n_{i}\right)$; briefly, this is true since both these differentials pull back to $\omega^{n}=\sum_{i=1}^{n} \pi_{i}^{*}(\omega)$ on $V^{n}$, by the quotient map $V^{n}=\prod_{i=1}^{r} V^{n_{i}} \rightarrow \prod_{i=1}^{r} V\left(n_{i}\right)$ (see [14, Lemma 4]). Thus Proposition 5 is proved.

Proposition 5 reduces the problem of computing the covector $\omega_{[n]}: T H_{[Z]} \rightarrow k$ to the case $Z=\operatorname{Spec}\left(O_{P} / I\right)$ is a 0 -dimensional closed subscheme of length $n$ concentrated at $P \in V\left(H=H_{V}^{n}\right.$ and $O_{P}=O$ is the local ring at $\left.P\right)$. In this case, $T H_{[Z]}=\operatorname{Hom}_{O}(I, O / I)$, by (10); thus a tangent vector $v \in T H_{[Z]}$ is given by an 
$O$-homomorphism $v: I \rightarrow O / I$. However, $v$ can also be given as a map $v: T=$ Spec $k[\varepsilon] \rightarrow H$ localized at $[Z]$; in order to compute using Theorem 2, we need to recall how these two descriptions of $v$ are related. The latter corresponds to a closed subscheme $W_{v} \subseteq V \times T$, etc., which is defined by an ideal $I_{v} \subseteq O \otimes_{k} k[\varepsilon]=O[\varepsilon]$, such that $M_{v}=O[\varepsilon] / I_{v}$ is free of dimension $n$ over $k[\varepsilon]$, and $I_{v} \otimes_{k[\varepsilon]} k=I$ (notation as in §4). If a tangent vector at $[Z]$ is given as an $O$-homomorphism $v$ : $I \rightarrow O / I$, then the proof of (9) essentially shows that the corresponding ideal $I_{v} \subset O[\varepsilon]=O \oplus \varepsilon O$ is obtained as follows:

$$
I_{v}=\{\alpha+\varepsilon \beta \mid \alpha \in I, \beta \in v(\alpha)\},
$$

where we view $v(\alpha) \in O / I$ as a coset of $I$ in $O$. Consequently,

$$
\alpha \equiv-\varepsilon \beta \quad\left(\bmod I_{v}\right),
$$

where $\alpha \in I$ and $\beta \in v(\alpha)$. We use this frequently below when making computations in $M_{v}$ (such as computing $\operatorname{tr}\left(g: M_{v} \rightarrow M_{v}\right)$ as required by Theorem 2).

Application 1. Let $C$ be an irreducible and nonsingular curve. We apply Theorems 1 and 2 to obtain a proof of (4). We begin with the key computation.

Let $P \in C, O_{P}=O$, the local ring at $P, t$ be a local parameter at $P, I=\left(t^{n}\right) \subset O$, and $Z=\operatorname{Spec} O / I$; thus $[Z]=(n P) \in C(n)=H_{C}^{n}$. It is easy to give a $k$-basis of $T C(n)_{(n P)}=\operatorname{Hom}_{O}(I, O / I)$; indeed, since $\left\{1, t, \ldots, t^{n-1}\right\}(\bmod I)$ is a $k$-basis of $O / I$, the $O$-homomorphisms determined by $t^{n} \mapsto t^{i}(0 \leqslant i \leqslant n-1)$ will do. Hence, any $v \in T C(n)_{(n P)}$ is determined by a unique choice of $n$ scalars $a_{i} \in k$, thus:

$$
v\left(t^{n}\right)=\left(a_{0}+a_{1} t+\cdots+a_{n-1} t^{n-1}\right) \in O / I .
$$

We now compute $\left(t^{r} d t\right)_{(n)}(v)$ for $0 \leqslant r \leqslant n-1$. By Theorem 1 ,

$$
\left(t^{r} d t\right)_{(n)}(v)=d t_{(n)} \circ \widehat{t^{r}}(v)=d t_{(n)}\left(t^{r} \cdot v\right),
$$

where $t^{r} \cdot v=v_{r} \in \operatorname{Hom}_{O}(I, O / I)$ is given by

$$
v_{r}\left(t^{n}\right)=\left(a_{0} t^{r}+a_{1} t^{r+1}+\cdots+a_{n-r-1} t^{n-1}\right) \in O / I,
$$

obtained by multiplying (22) through by $t^{r}$. By Theorem 2 ,

$$
d t_{(n)}\left(v_{r}\right)=C_{\varepsilon}\left(\operatorname{tr}\left(t: M_{v_{r}} \rightarrow M_{v_{r}}\right)\right)
$$

to compute the trace, we use the $k[\varepsilon]$-basis of $M_{v_{r}}=O[\varepsilon] / I_{v_{r}}$ given by $\left\{1, t, \ldots, t^{n-1}\right\}$ (Nakayama's lemma). We have that

$$
\begin{gathered}
t \cdot t^{i}=t^{i+1} \quad \text { for } 0 \leqslant i \leqslant n-2, \quad \text { and } \\
t \cdot t^{n-1}=t^{n}=-\varepsilon\left(a_{0} t^{r}+a_{1} t^{r+1}+\cdots+a_{n-r-1} t^{n-1}\right),
\end{gathered}
$$

where the last equality results from (21) and (23). The trace is $-\varepsilon \cdot a_{n-r-1}$, which has $\varepsilon$-coefficient $-a_{n-r-1}$; consequently, for $v$ as in (22),

$$
\left(t^{r} d t\right)_{(n)}(v)=d t_{(n)}\left(v_{r}\right)=-a_{n-r-1}, \quad 0 \leqslant r \leqslant n-1 .
$$

We may now prove (4); in view of Proposition 5, it is sufficient to prove the following statement:

(25) $\omega_{(n)}$ is zero at $(n P) \in C(n) \Leftrightarrow(\omega)>n P$, where $(\omega)$ is the divisor of $\omega$ on $C$. 
Proof. We use the notation established above. Locally at $P$ we write $\omega=h d t=$ $\left(b_{0}+b_{1} t+\cdots+b_{n-1} t^{n-1}+h_{n}\right) d t$, where $h_{n} \in\left(t^{n}\right)$; using the linearity of $\omega \mapsto \omega_{(n)}$ and Theorem 1 we obtain

$$
\omega_{(n)}=\sum_{i=0}^{n-1} b_{i}\left(t^{i} d t\right)_{(n)}+d t_{(n)} \circ \hat{h}_{n} \quad \text { locally at }(n P) \text {. }
$$

Now choose $v \in T C(n)_{(n P)}=\operatorname{Hom}_{O}(I, O / I)$ as in (22). Then

$$
\begin{aligned}
\omega_{(n)}(v) & =\sum_{i=0}^{n-1} b_{i}\left(t^{i} d t\right)_{(n)}(v)+d t_{(n)} \circ \hat{h}_{n}(v) \\
& =-\sum_{i=0}^{n-1} b_{i} \cdot a_{n-i-1}+0,
\end{aligned}
$$

where the first term results from (24) and the second term is zero since multiplication by $h_{n} \in\left(t^{n}\right)=I$ kills the tangent space (as in Corollary 2 of Theorem 1). Therefore,

$$
\begin{aligned}
\omega_{(n)} \text { is zero at }(n P) \in C(n) & \Leftrightarrow \omega_{(n)}(v)=0 \text { for all } v \in T C(n)_{(n P)} \\
& \Leftrightarrow-\sum_{i=0}^{n-1} b_{i} \cdot a_{n-i-1}=0 \text { for all choices of the } a \text { 's } \\
& \Leftrightarrow b_{0}=b_{1}=\cdots=b_{n-1}=0 \\
& \Leftrightarrow h \in\left(t^{n}\right) \\
& \Leftrightarrow(\omega)>n P \text { on } C,
\end{aligned}
$$

which completes the proof.

We set the stage for our next application by proving that if $C$ is a complete nonsingular curve of genus $g$ and $A$ is its Albanese (= Jacobian) variety, then

(26) for $n \geqslant 2 g-1$, the map $\varphi_{n}=f_{n}: C(n) \rightarrow A$ is smooth.

Indeed, by (2), it is enough to show that the pullback $\varphi_{n}^{*}\left(\omega_{A}\right)$ has no zeros on $C(n)$, where $\omega_{A}$ is an arbitrary nonzero holomorphic 1 -form on $A$. But by (3), $\varphi_{n}^{*}\left(\omega_{A}\right)=\omega_{(n)}$ is the symmetrization of the nonzero 1-form $\omega=f^{*}\left(\omega_{A}\right)$ on $C$, and by (4), $\omega_{(n)}$ is zero at $\left(\sum_{i=1}^{r} n_{i} P_{i}\right) \in C(n) \Leftrightarrow(\omega)>\sum_{i=1}^{r} n_{i} P_{i}$. But $(\omega)$ has degree $2 g-2$, so for $n \geqslant 2 g-1, \omega_{(n)}$ can have no zeros, and (26) follows.

Application 2. We show that no statement analogous to (26) can hold for the map $\varphi_{n}: H_{X}^{n} \rightarrow A$ for surfaces $X$ : in general, $\varphi_{n}$ will not be smooth for $n \gg 0$. More precisely, we have

THEOREM 3. Let $X$ be an irreducible and nonsingular surface for which the Albanese mapping $f: X \rightarrow A$ is not smooth. Then for $n=r(r+1) / 2, r>0$, the mapping $\varphi_{n}$ : $H_{X}^{n} \rightarrow A$ is not smooth.

REMARK. The hypothesis holds, for example, whenever $\operatorname{dim} A \geqslant 3$.

The proof of Theorem 3 is based on a calculation requiring only Theorem 1, which we state as Proposition 6 . Let $X$ be an irreducible and nonsingular surface, $\omega$ a holomorphic 1-form on $X$, and $x, y$ local parameters at $P \in X$. Note that $M_{P}^{r}$ has 
colength $r(r+1) / 2$ in the local ring $O_{P}$ (since the classes $\left(\bmod M_{p}^{r}\right.$ ) of $1, x, y, x^{2}, \ldots, x^{r-1}, x^{r-2} y, \ldots, y^{r-1}$ form a basis of $O_{P} / M_{P}^{r}$ over the ground field $k$ ), and let $Z_{r}=\operatorname{Spec} O_{P} / M_{P}^{r}$, a 0-dimensional closed subscheme of length $r(r+1) / 2$ concentrated at $P$.

Proposition 6. If $\omega$ is zero at $P \in X$, then $\omega_{[n]}$ is zero at $\left[Z_{r}\right] \in H_{X}^{n}$ for all $r>0$, $n=r(r+1) / 2$.

Proof. Since $\omega$ is zero at $P$, we may write $\omega=h_{1} d x+h_{2} d y$ locally at $P$, with $h_{1}, h_{2} \in M_{p}$. By Theorem 1

$$
\omega_{[n]}=d x_{[n]} \circ \hat{h}_{1}+d y_{[n]} \circ \hat{h}_{2}
$$

in a neighborhood of $\left[Z_{r}\right] \in H_{X}^{n}$; it therefore suffices to prove that $\hat{h}_{1}$ and $\hat{h}_{2}$ are each the zero map on $T H_{\left[Z_{r}\right]}=\operatorname{Hom}_{O_{P}}\left(M_{P}^{r}, O_{P} / M_{P}^{r}\right)$, which follows from the

LeMma. For any $h \in M_{P}$ and $r>0$, multiplication by $h$ on $\operatorname{Hom}_{O_{P}}\left(M_{P}^{r}, O_{P} / M_{P}^{r}\right)$ is the zero map.

Proof. In effect, we must prove that the image of any $v \in \operatorname{Hom}_{O_{P}}\left(M_{P}^{r}, O_{P} / M_{P}^{r}\right)$ lies in $M_{P}^{r-1} / M_{P}^{r}$, or equivalently, that

$$
\operatorname{Hom}_{O_{P}}\left(M_{P}^{r}, M_{P}^{r-1} / M_{P}^{r}\right)=\operatorname{Hom}_{O_{P}}\left(M_{P}^{r}, O_{P} / M_{P}^{r}\right) .
$$

Since the LHS is a subspace of the RHS, it suffices to prove that $\operatorname{dim}_{k}($ LHS $)=2 n$, which is the dimension of the RHS.

To do this, note that $M_{P}^{r}$ has $r+1$ generators $s_{0}=x^{r}, s_{1}=x^{r-1} y, \ldots, s_{r}=y^{r}$, which give a $k$-basis of $M_{P}^{r} / M_{P}^{r+1}$, and likewise $M_{P}^{r-1} / M_{P}^{r}$ has a $k$-basis of $r$ elements given by $t_{0}=x^{r-1}, t_{1}=x^{r-2} y, \ldots, t_{r-1}=y^{r-1}$; moreover, $r(r+1)=$ $w(r(r+1) / 2)=2 n$. One checks easily that given any choice of $2 n$ scalars $a_{i j} \in k$ $(0 \leqslant i \leqslant r, 0 \leqslant j \leqslant r-1)$, we get a well-defined $O_{P}$-linear map $v: M_{P}^{r} \rightarrow M_{P}^{r-1} / M_{P}^{r}$ by setting $v\left(s_{i}\right)=\sum_{j=0}^{r-1} a_{i j} t_{j}, 0 \leqslant i \leqslant r$. Thus $\operatorname{dim}_{k}($ LHS $)=2 n$, which proves the lemma and the proposition.

Proof of Theorem 3. By hypothesis, $f: X \rightarrow A$ is not smooth, say at the point $P \in X$; by (2) applied to $f=\varphi_{1}$, this means that for some nonzero 1-form $\omega_{A}$ on $A$, the pullback $f^{*}\left(\omega_{A}\right)=\omega$ has a zero at $P$. Let $Z_{r}=\operatorname{Spec} O_{P} / M_{P}^{r}$ as above. Then (2), (3), and Proposition 6 combine to prove that

(27) $\left[Z_{r}\right] \in H_{X}^{n}$ is a singularity of $\varphi_{n}$, where $n=r(r+1) / 2, r>0$.

Letting $r=1,2,3, \ldots$, we obtain the desired conclusion.

REMARK. It is shown in [6, Theorem 3] that for $n \gg 0$, all the fibers of $\varphi_{n}$ have the minimal dimension $2 n-q$. It follows that $\varphi_{n}$ is flat for $n \gg 0$, since any dominating map from a Cohen-Macaulay (in particular, nonsingular) variety to a nonsingular variety is flat wherever the fibers have the minimal dimension $[1, \mathrm{~V}, 3.5]$. Since the singularities of a flat map are singular points of their (scheme-theoretic) fibers [1, VII, 1.8], we see that the singularities of $\varphi_{n}$ for $n \gg 0$ (for example, those given by (27)) are singular points of their fibers.

According to Theorem 3

(28) $\varphi_{n}: H_{X}^{n} \rightarrow A$ is smooth for $n \gg 0 \Rightarrow$ the Albanese mapping $f: X \rightarrow A$ is smooth, where $X$ is an irreducible and nonsingular surface. 
Our next goal is to prove the converse of (28) holds if the base field is $k=\mathbf{C}$ (Theorem 4). In characteristic $p>0$, the converse is false; a counterexample will be given in Application 3 below. For the proof of Theorem 4 we will need

Proposition 7. Let $X$ be an irreducible and nonsingular surface in characteristic 0 , $\omega$ a holomorphic 1-form on $X$, and $Z$ a 0-dimensional closed subscheme of length $n$ on $X$. If $\omega_{[n]}$ is zero at $[Z] \in H_{X}^{n}$, then $\omega$ is zero on $\operatorname{Supp} Z \subset X$.

Proof. In view of Proposition 5, we may assume that Supp $Z=\{P\} \subset X$.

We use the theory of induced differentials developed in [11 and 14]. Given any map $d: S \rightarrow X(n)$ having domain a nonsingular variety, the symmetrized differential $\omega_{(n)}$ induces a holomorphic differential denoted $d^{*}\left(\omega_{(n)}\right)$ on $S$ (which is defined even if the image of $S$ lies in the singular locus of $X(n)$ ). The induced differential behaves functorially. If $d$ is dominating, then the induced differential is just the usual pullback of $\omega_{(n)}$ as a rational differential.

Consider the diagonal map $d=d_{n}: X \rightarrow X(n)$ given by $d(Q)=(n Q)$ for all $Q \in X$; by factoring $d$ through $X^{n}$ in the obvious way and using the functorial behavior of the induced differential, we see that $d^{*}\left(\omega_{(n)}\right)=n \cdot \omega$ on $X$.

Now consider the diagram

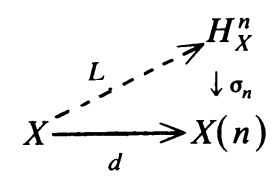

where the dashed arrow has yet to be defined. If there exists a dashed arrow $L$ defined in a neighborhood of $P$, which maps $P$ to $[Z]$ and renders the diagram commutative (where defined), we can conclude that

$$
n \omega=d^{*}\left(\omega_{(n)}\right)=L^{*} \sigma_{n}^{*}\left(\omega_{(n)}\right)=L^{*}\left(\omega_{[n]}\right)
$$

holds near $P$. But by hypothesis $\omega_{[n]}$ is zero at $[Z]$, hence its pullback $n \cdot \omega$ is zero at the preimage of $[Z]$, which is $P$. Since the characteristic is 0 , it follows that $\omega$ is zero at $P$, as desired.

To complete the proof, therefore, it suffices to construct a lifting $L: U \rightarrow H$ as described above, defined on a neighborhood $U$ of $P \in X$. By the universal property of $H, L$ will correspond to a family of subschemes of $X$, flat and finite of length $n$ over $U$, such that the subscheme over $Q \in U$ is supported only at $Q$ (to ensure commutativity of the diagram), and such that the subscheme lying over $P$ is $Z$.

First consider the case $X=\mathbf{A}^{2}=\operatorname{Spec} k[x, y]$ is the affine plane, $P=(0,0)$, and $Z$ is defined by the ideal $I=\left(g_{1}(x, y), \ldots, g_{r}(x, y)\right) \subset k[x, y]$. Consider the closed subscheme $W \subset \mathbf{A}^{2} \times \mathbf{A}^{2}$ defined by the ideal

$$
I^{\prime}=\left(g_{1}\left(x_{1}-x_{2}, y_{1}-y_{2}\right), \ldots, g_{r}\left(x_{1}-x_{2}, y_{1}-y_{2}\right)\right),
$$

where $\left(x_{1}, y_{1} ; x_{2}, y_{2}\right)$ is a coordinate system on $\mathbf{A}^{2} \times \mathbf{A}^{2}$. Map $W$ to $\mathbf{A}^{2}$ by the second projection; we claim that in this way $W$ is a flat and finite family of subschemes of length $n$ over $\mathbf{A}^{2}$. 
Note that the fiber of $p r_{2}: W \rightarrow \mathbf{A}^{2}$ over $(\alpha, \beta)$ is the closed subscheme $W(\alpha, \beta)$ of the first factor $\mathbf{A}^{2}$ defined by the ideal

$$
I_{(\alpha, \beta)}=\left(g_{1}\left(x_{1}-\alpha, y_{1}-\beta\right), \ldots, g_{r}\left(x_{1}-\alpha, y_{1}-\beta\right)\right) ;
$$

since this is the pullback of $I=I_{(0,0)}$ by an isomorphism (translation) of $\mathbf{A}^{2}$, we see that $W_{(\alpha, \beta)}$ is supported at $(\alpha, \beta)$ and has length $n$ there. This proves that the support of $W$ is the diagonal of $\mathbf{A}^{2} \times \mathbf{A}^{2}$, hence $p r_{2}: W \rightarrow \mathbf{A}^{2}$ is finite, and since the length of $W_{(\alpha, \beta)}$ is always $n, p r_{2}: W \rightarrow \mathbf{A}^{2}$ is flat as well, by [13, Proposition 8, p. 44]. Finally, since $W_{(0,0)}=Z$, the lifting $L: \mathbf{A}^{2} \rightarrow H_{A^{2}}^{n}$ exists as required in this case.

In the general case, since $X$ is nonsingular; there exists a rational map $\beta: X \rightarrow \mathbf{A}^{2}$ which carries $P$ to $(0,0)$ and is étale in some neighborhood $U$ of $P$. Let $Z^{\prime}$ be the closed subscheme of length $n$ supported at $(0,0) \in \mathbf{A}^{2}$ that corresponds to $Z$ under the local analytic isomorphism $\hat{O}_{\mathrm{A}^{2},(0,0)} \stackrel{\sim}{\rightarrow} \hat{O}_{X, P}$. Form the closed subscheme $W \subseteq \mathbf{A}^{2}$ $\times \mathbf{A}^{2}$ as above, starting with $Z^{\prime}$ at $(0,0)$, and let $W^{*}$ be the pullback:

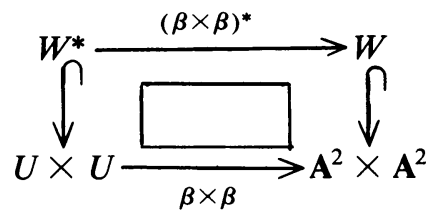

The support of $W^{*}$ is the pullback of the diagonal in $\mathbf{A}^{2} \times \mathbf{A}^{2}$; since $(\beta \times \beta)^{*}$ : $\operatorname{Supp} W^{*} \rightarrow \operatorname{Supp} W$ is étale, we have that $\operatorname{Supp} W^{*}$ is nonsingular, and therefore is the disjoint union of its connected (irreducible) components, one of which is the diagonal of $U \times U$. Moreover, $W^{*}$ has no embedded components, since it is étale over $W$ and $W$ has no embedded components (being flat over $\mathbf{A}^{2}$ ). Thus $W^{*}$ is the disjoint union of its minimal primary components, each of which is étale over $W$.

Let $W_{L}$ be the primary component of $W^{*}$ supported on the diagonal of $U \times U$, and map $W_{L}$ to $U$ by the second projection. As above it follows that $p r_{2}: W_{L} \rightarrow U$ is finite. To see $p r_{2}: W_{L} \rightarrow U$ is flat, we must check that the fibers all have the same length $n$, but this follows since these fibers are étale over the corresponding fibers of $p r_{2}: W \rightarrow \mathbf{A}^{2}$, all of which have length $n$. Finally, by construction, the fiber of $W_{L}$ over $P$ is $Z$, so the lifting $L$ exists as required in the general case, and the proposition is proved.

THEOREM 4. Let $X$ be an irreducible and nonsingular surface $/ \mathbf{C}$, with $q=\operatorname{dim} A>0$. Then the following are equivalent:

(i) $\varphi_{n}: H_{X}^{n} \rightarrow A$ is smooth for $n \gg 0$;

(ii) the Albanese mapping $\varphi_{1}=f: X \rightarrow A$ is smooth;

(iii) every nonzero holomorphic 1-form $\omega=f^{*}\left(\omega_{A}\right)$ has no zeros on $X\left(0 \neq \omega_{A} \in\right.$ $\left.\Gamma\left(\Omega_{A}^{1}\right)\right)$

(iv) $\varphi_{n}: H_{X}^{n} \rightarrow A$ is smooth for all $n>0$.

ProOF. (i) $\Rightarrow$ (ii). This is (28).

(ii) $\Leftrightarrow$ (iii). This follows from (2) applied to $\varphi_{1}=f: X \rightarrow A$.

(iii) $\Rightarrow$ (iv). Suppose (iii) holds, and that for some $n, \varphi_{n}: H_{X}^{n} \rightarrow A$ has a singularity at $[Z]$. Then, by (2), $\varphi_{n}^{*}\left(\omega_{A}\right)$ is zero at [Z] for some nonzero holomorphic 1-form $\omega_{A}$ 
on $A$, and by (3), $\varphi_{n}^{*}\left(\omega_{A}\right)=\omega_{[n]}$, where $\omega=f^{*}\left(\omega_{A}\right)$. Proposition 7 implies that $\omega$ is zero on Supp $Z$, but (iii) says that $\omega$ can have no zeros, contradiction.

(iv) $\Rightarrow$ (i). Clear.

REMARK. If $X$ is complete, then (as remarked in $\S 1$ ) $f^{*}: \Gamma\left(\Omega_{A}^{1}\right) \rightarrow \Gamma\left(\Omega_{X}^{1}\right)$ will be an isomorphism. Then (iii) reads: every nonzero holomorphic 1 -form $\omega$ on $X$ has no zeros on $X$.

The class of surfaces satisfying the conditions of the theorem is quite restricted:

Proposition 8. Let $X$ be a complete nonsingular surface / $\mathbf{C}$ with irregularity $q>0$, satisfying the conditions (i)-(iv) of Theorem 4. Then either $X$ is an abelian surface or $X$ has the invariants $q=1, p_{g}=0$.

RemarK. Clearly every abelian surface/C will satisfy the conditions of the theorem. Examples of surfaces satisfying (i)-(iv) in the latter class include $X=\mathbf{P}^{1} \times$ $E$, where $E$ is an elliptic curve, and $X=E(2)$ (use (4) to show (iii) holds for $E(2)$ ).

Proof. (I am endebted to the referee for this argument.) By (ii), $f: X \rightarrow A$ is smooth, therefore $0<q \leqslant 2$. If $q=2$, then $X$ is an etale cover of an abelian surface, and consequently is itself an abelian surface [12, p. 167]. If $q=1$, then a nowhere zero 1-form $\omega$ on $X$ gives an exact sequence

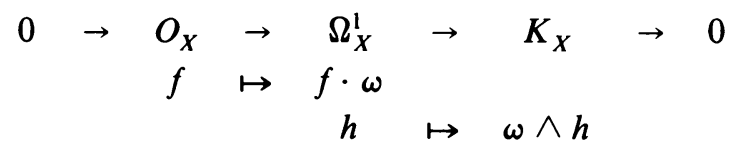

of sheaves on $X$. From the long exact cohomology sequence, duality, and Hodge theory, one finds $h^{1,1}=2-2 p_{g}>0 \Rightarrow p_{g}=0$.

Application 3. Theorem 4 implies that $\varphi_{n}: H_{A}^{n} \rightarrow A$ is smooth for all $n>0$ if $A$ is an abelian surface/C. In this application we study $\varphi_{n}$ for abelian surfaces defined over algebraically closed fields $k$ of any characteristic. We give an easy proof that $\varphi_{n}$ is smooth for $n$ prime to the characteristic, and find a counterexample to smoothness for all $n$.

TheOREM 5. Let $A$ be an abelian surface. If char $k=p \nmid n$, then the map $\varphi_{n}$ : $H_{A}^{n} \rightarrow A$ is smooth.

Proof. Let $[Z] \in H_{A}^{n}=H$ and let $\varphi_{n}([Z])=a \in A$; by (1), we must prove that the differential map $D \varphi_{n}: T H_{[Z]} \rightarrow T A_{a}$ is surjective. To do this, we first observe that $A$ acts on $H_{A}^{n}$ in a natural way: translation by $b \in A$ on $H_{A}^{n}$ carries [Z] to [ $Z_{b}$ ], where $\left[Z_{b}\right]$ is defined by pullback as shown:

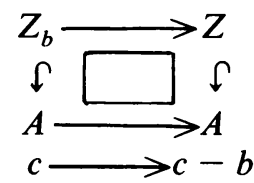

(For details concerning this action, see [6, Part 2].) In particular, if $\sigma_{n}([Z])=\left(a_{1}\right.$ $\left.+\cdots+a_{n}\right) \in A(n)$, then $\sigma_{n}\left(\left[Z_{b}\right]\right)=\left(\left(a_{1}+b\right)+\cdots+\left(a_{n}+b\right)\right)\left(\left(a_{i}+b\right)\right.$ is the sum in $A$ ); consequently

$$
\varphi_{n}\left(\left[Z_{b}\right]\right)=\varphi_{n}([Z])+n b=a+n b .
$$


Consider now the following commutative diagrams of maps and associated differential maps

$\begin{array}{cccccc} & & H_{A}^{n} & & & T H_{[Z]} \\ & \tau \nearrow & \downarrow \varphi_{n} & & & \\ A & \rightarrow & A & & D \tau \nearrow & \downarrow D \varphi_{n} \\ b & \mapsto & a+n b & T A_{e} & \rightarrow & T A_{a}\end{array}$

where $e \in A$ is the identity and $\tau$ is the map $b \mapsto\left[Z_{b}\right]$ (whose image is the orbit of $[Z]$ ). Since the isogeny of multiplication by $n$ on $A$ is étale whenever $p \nmid n$ (our hypothesis), we have that the horizontal arrow in the right-hand digram is surjective. But this forces the vertical arrow $D \varphi_{n}$ to be surjective as well, which proves the theorem.

Let char $k=p>0$. We will show that for any (irregular) irreducible and nonsingular surface $X$ and any $n=m^{2} \equiv 0(\bmod p)$, the map $\varphi_{n}: H_{X}^{n} \rightarrow A$ has singularities (Theorem 6). To produce these singularities, we need the next proposition, whose proof is an explicit computation based on Theorems 1 and 2.

Let $O_{P}=O$ be the local ring of $P \in X, x, y$ local parameters at $P, I=\left(x^{m}, y^{m}\right)$ $\subset O$, where $p \mid m$, and $Z=\operatorname{Spec} O / I$. The colength of $I$ is $n=m^{2}$, as may be seen by counting the monomials $x^{i} y^{j}(0 \leqslant i, j \leqslant m-1)$, who residues $(\bmod I)$ form a $k$-basis of $O / I$. The point $[Z] \in H_{X}^{n}=H$ has a remarkable property, given by

Proposition 9. The symmetrization $\omega_{[n]}$ of any holomorphic 1-form $\omega$ on $X$ is zero at $[Z]=\left[\operatorname{Spec} O_{P} /\left(x^{m}, y^{m}\right)\right]$, where $n=m^{2} \equiv 0(\bmod p)$.

Proof. Since $\omega=h_{1} d x+h_{2} d y$ locally at $P$, its symmetrization $\omega_{[n]}$ is equal to $d x_{[n]} \circ \hat{h}_{1}+d y_{[n]} \circ \hat{h}_{2}$ near [Z] by Theorem 1, so it suffices to prove that $d x_{[n]}$ and $d y_{[n]}$ are zero at $P$; by symmetry, it is enough to do this for $d x_{[n]}$. We will use Theorem 2 to show explicitly that $d x_{[n]}(v)=0$ for all $v \in T H_{[Z]}$.

We first note that a basis of $T H_{[Z]}=\operatorname{Hom}_{O}(I, O / I)$ is given by the $O$-homomorphisms determined by $x^{m} \mapsto x^{r_{1}} y^{s_{1}}$ and $y^{m} \mapsto x^{r_{2}} y^{s_{2}} \quad\left(0 \leqslant r_{1}, s_{1}, r_{2}, s_{2} \leqslant m-1\right)$ (count dimensions); hence, any $v \in T H_{[Z]}$ is uniquely specified by a choice of $2 n$ scalars $a_{r, s}, b_{r, s} \in k$, thus ( $\sum$ over $\left.0 \leqslant r, s \leqslant m-1\right)$ :

$$
\begin{aligned}
& v\left(x^{m}\right)=\sum a_{r, s} x^{r} y^{s} \in O / I, \\
& v\left(y^{m}\right)=\sum b_{r, s} x^{r} y^{s} \in O / I .
\end{aligned}
$$

By Theorem 2, $d x_{[n]}(v)=C_{\varepsilon}\left(\operatorname{tr}\left(x: M_{v} \rightarrow M_{v}\right)\right)$; to compute the trace, we use the $k[\varepsilon]$-basis $\left\{x^{i} y^{j} \mid 0 \leqslant i, j \leqslant m-1\right\}\left(\bmod I_{v}\right)$ of $M_{v}=O[\varepsilon] / I_{v}$ (Nakayama's lemma again). We have that

$$
x \cdot\left(x^{i} y^{j}\right)=x^{i+1} y^{j} \text { for } 0 \leqslant i \leqslant m-2,0 \leqslant j \leqslant m-1,
$$

which contributes nothing to the trace, and

$$
x \cdot\left(x^{m-1} y^{j}\right)=x^{m} y^{j}=-\varepsilon\left(\sum a_{r, s} x^{r} y^{s}\right) \cdot y^{j}=-\varepsilon\left(\sum a_{r, s} x^{r} y^{s+j}\right),
$$

where the next-to-last equality results from (21) and (29). Whenever $s+j \geqslant m$, we have that $y^{s+j}=-\varepsilon\left(\sum b_{r^{\prime}, s^{\prime}} x^{r^{\prime}} y^{s^{\prime}}\right) \cdot y^{s+j-m}$ (again by (21) and (29)); since $\varepsilon^{2}=0$, the terms where $s+j \geqslant m$ drop out of the sum. From the remaining terms, we get a 
contribution to the trace when $x^{m-1} y^{j}=x^{r} y^{s+j}(\Rightarrow r=m-1$ and $s=0)$, and this contribution is $-\varepsilon a_{m-1,0}$. Since we get this contribution $m$ times $(0 \leqslant j \leqslant m-1)$, the trace equals $-m \varepsilon a_{m-1,0}$, which has $\varepsilon$-coefficient $-m a_{m-1,0}$. But this is zero, since we have assumed that $p \mid m$, so the proposition is proved.

THEOREM 6. Let $X$ be any irreducible and nonsingular irregular surface defined over an algebraically closed field $k$ of characteristic $p>0$. Then for any $n=m^{2} \equiv 0$ $(\bmod p)$, the mapping $\varphi_{n}: H_{X}^{n} \rightarrow A$ has singularities.

Proof. Let $P$ be any point of $X$, and let $Z=\operatorname{Spec} O_{P} /\left(x^{m}, y^{m}\right)$ as above. Then (2), (3), and Proposition 9 imply immediately that

(30) $[Z]=\left[\operatorname{Spec} O_{P} /\left(x^{m}, y^{m}\right)\right]$ is a singularity of the mapping $\varphi_{n}: H_{X}^{n} \rightarrow A$, where $n=m^{2} \equiv 0(\bmod p)$, whence the theorem.

Specializing to the case of an abelian surface $X=A$, we conclude that $\varphi_{n}: H_{A}^{n} \rightarrow A$ is not smooth for $n$ as in the theorem; this gives the counterexample to smoothness of $\varphi_{n}$ for all $n$ promised above. It also gives a counterexample to the converse of (28) in characteristic $p$ : the Albanese mapping $f: A \rightarrow A$ is smooth, but $\varphi_{n}$ is not smooth for $n \gg 0$. But there is more to say. We first note that every fiber of $\varphi_{n}$ has singularities of the type given by (30): given $a \in A$, choose $c \in A$ such that $n c=a$ (divisibility of $A$ ), and form the subscheme $Z=\operatorname{Spec} O_{c} /\left(x_{m}, y_{m}\right)$ as above; then $[Z]$ is a singularity of $\varphi_{n}$ lying over $\varphi_{n}([Z])=n c=a$. This exemplifies the well-known failure of Sard's theorem in char $p$ (every $a \in A$ is a "critical value" of $\varphi_{n}$ ).

Furthermore, since the fibers of $\varphi_{n}$ all have the minimal dimension $2 n-q$ [6, Theorem 4], our earlier argument (remark following proof of Theorem 3) shows that the singularities of $\varphi_{n}$ are singular points of their fibers. By contrast, it is proved in [6, Theorem 6, Corollary 1] that if $p \nmid n$, then the fibers of $\varphi_{n}: H_{A}^{n} \rightarrow A$ are nonsingular varieties (this follows from Theorem 5) which desingularize the corresponding fibers of $f_{n}: A(n) \rightarrow A$; the case where $p \mid n$ is not settled by the method used there. We now see that this desingularization fails if $p \mid n$.

ApPlication 4. Our goal here is to formulate and prove a natural generalization of (4) for curves to the case of surfaces which appears as Theorem 8 below. We first rephrase (25), the local version of (4), in the language of jets.

Let $Y$ be a nonsingular variety of dimension $\gamma$, and $\Omega_{Y, y}^{1}$ the $O_{Y, y}$-module of germs of holomorphic 1-forms at $y \in Y$. We say that two holomorphic 1-forms $\omega_{1}$ and $\omega_{2}$ on $Y$ have the same $m$-jet at $y \Leftrightarrow$ the germ of $\omega_{1}-\omega_{2} \in M_{y}^{m+1} \cdot \Omega_{Y, y}^{1}$. In other words, if we write $\omega_{1}=h_{1,1} d x_{1}+\cdots+h_{1, r} d x_{r}$ and $\omega_{2}=h_{2,1} d x_{1}+\cdots+h_{2, r} d x_{r}$ using the local parameters $x_{1}, \ldots, x_{r}$ at $y$, then the power series expansions of $h_{1, i}$ and $h_{2, i}$ agree to order $m$ for $1 \leqslant i \leqslant r$. In this language, we see that (25) is equivalent to the following statement:

(31) $\omega_{(n)}$ is zero at $(n P) \in C(n) \Leftrightarrow$ the $(n-1)$-jet of $\omega$ is zero at $P \in C$, where $C$ is an irreducible and nonsingular curve and $\omega$ is a holomorphic l-form on $C$.

We seek to generalize (31) to surfaces; the first step is another explicit computation using Theorems 1 and 2, which we state as Proposition 10 . Let $X$ be an irreducible and nonsingular surface, $O_{P}=O$ the local ring at $P \in X, x, y$ local parameters at $P, I=\left(x^{n}, y\right) \subset O$, and $Z=\operatorname{Spec} O / I$; thus $Z$ has length $n$ 
$\left(\left\{1, x, \ldots, x^{n-1}\right\}(\bmod I)\right.$ is a basis of $\left.O / I\right)$ and is concentrated at $P$. Let $\omega=h_{1} d x$ $+h_{2} d y$ be a holomorphic 1-form on $X$. Expand $h_{1}$ and $h_{2}$ in power series $\left(\Sigma=\Sigma_{0<k, l}\right)$ :

$$
h_{1}=\sum b_{k, l} x^{k} y^{l}, \quad h_{2}=\sum c_{k, l} x^{k} y^{l}
$$

Proposition 10. The symmetrized differential $\omega_{[n]}$ is zero at $[Z] \in H_{X}^{n} \Leftrightarrow b_{0,0}=$ $b_{1,0}=\cdots=b_{n-1,0}=0$ and $n \cdot c_{0,0}=0\left(\Rightarrow c_{0,0}=0\right.$ if $\left.\operatorname{char} k=p \nmid n\right)$.

Proof. We first note that a basis of $T H_{[Z]}=\operatorname{Hom}_{o}(I, O / I)$ is given by the $O$-homomorphisms determined by $x^{n} \mapsto x^{i}$ and $y \mapsto x^{j}$, where $0 \leqslant i, j \leqslant n-1$ (count dimensions); hence, any element $v \in T H_{[Z]}$ is uniquely specified by a choice of $2 n$ scalars $\alpha_{r}, \beta_{r} \in k$, thus:

$$
\begin{aligned}
& v\left(x^{n}\right)=\sum_{r=0}^{n-1} \alpha_{r} x^{r} \in O / I, \\
& v(y)=\sum_{r=0}^{n-1} \beta_{r} x^{r} \in O / I .
\end{aligned}
$$

We now compute $\omega_{[n]}(v)$ in terms of the $\alpha$ 's, $\beta$ 's, $b$ 's, and $c$ 's; by requiring that the result be zero for all choices of the $\alpha$ 's and $\beta$ 's (all $v \in T H_{[Z]}$ ), we will obtain the conditions stated in the proposition.

By Theorem 1,

$$
\begin{aligned}
\omega_{[n]}(v) & =d x_{[n]} \circ \hat{h}_{1}(v)+d y_{[n]} \circ \hat{h}_{2}(v) \\
& =d x_{[n]}\left(\left(\sum b_{k, l} x^{k} y^{l}\right) \cdot v\right)+d y_{[n]}\left(\left(\sum c_{k, l} x^{k} y^{l}\right) \cdot v\right) \\
& =d x_{[n]}\left(\left(\sum_{k=0}^{n-1} b_{k, 0} x^{k}\right) \cdot v\right)+d y_{[n]}\left(\left(\sum_{k=0}^{n-1} c_{k, 0} x^{k}\right) \cdot v\right),
\end{aligned}
$$

where the terms having $x$-degree $\geqslant n$ or $y$-degree $\geqslant 1$ have dropped out since $x^{n} \equiv y \equiv 0(\bmod I)$. Let $v_{1}=\left(\sum_{k=0}^{n-1} b_{k, 0} x^{k}\right) \cdot v$ and $v_{2}=\left(\sum_{k=0}^{n-1} c_{k, 0} x^{k}\right) \cdot v$; using (32) and multiplying out, we see that $v_{1}$ and $v_{2}$ are given as $O$-homomorphisms by

$$
\begin{aligned}
& v_{1}\left(x^{n}\right)=\sum_{s=0}^{n-1}\left(\sum_{r+k=s} b_{k, 0} \alpha_{r}\right) \cdot x^{s} \in O / I, \\
& v_{1}(y)=\sum_{s=0}^{n-1}\left(\sum_{r+k=s} b_{k, 0} \beta_{r}\right) \cdot x^{s} \in O / I, \\
& v_{2}\left(x^{n}\right)=\sum_{s=0}^{n-1}\left(\sum_{r+k=s} c_{k, 0} \alpha_{r}\right) x^{s} \in O / I, \\
& v_{2}(y)=\sum_{s=0}^{n-1}\left(\sum_{r+k=s} c_{k, 0} \beta_{r}\right) x^{s} \in O / I .
\end{aligned}
$$

We now compute $d x_{[n]}\left(v_{1}\right)$ and $d y_{[n]}\left(v_{2}\right)$.

$d x_{[n]}\left(v_{1}\right)$. By Theorem 2, $d x_{[n]}\left(v_{1}\right)=C_{\varepsilon}\left(\operatorname{tr}\left(x: M_{v_{1}} \rightarrow M_{v_{1}}\right)\right)$; to compute the trace, we use the $k[\varepsilon]$-basis $\left\{x^{i} \mid 0 \leqslant i \leqslant n-1\right\}\left(\bmod I_{v_{1}}\right)$ of $M_{v_{1}}=O[\varepsilon] / I_{v_{1}}$. We have that

$$
x \cdot x^{i}=x^{i+1} \quad \text { for } 0 \leqslant i \leqslant n-2 \text {, }
$$


which contributes nothing to the trace, and

$$
x \cdot x^{n-1}=x^{n}=-\varepsilon\left(\sum_{s=0}^{n-1}\left(\sum_{r+k=s} b_{k, 0} \alpha_{r}\right) \cdot x^{s}\right),
$$

where the last equality results from (21) and (33). The sole contribution to the trace comes from the last expression when $s=n-1$, and this contribution has $\varepsilon$-coefficient

$$
d x_{[n]}\left(v_{1}\right)=-\sum_{r+k=n-1} b_{k, 0} \alpha_{r} .
$$

$d y_{[n]}\left(v_{2}\right)$. By Theorem 2, $d y_{[n]}\left(v_{2}\right)=C_{\varepsilon}\left(\operatorname{tr}\left(y: M_{v_{2}} \rightarrow M_{v_{2}}\right)\right)$; we use the $k[\varepsilon]$-basis $\left\{x^{i} \mid 0 \leqslant i \leqslant n-1\right\}\left(\bmod I_{v_{2}}\right)$ to compute the trace.

Before beginning, we remark that

$$
y \equiv-\varepsilon\left(\sum_{s=0}^{n-1}\left(\sum_{r+k=s} c_{k, 0} \beta_{r}\right) x^{s}\right) \quad\left(\bmod I_{v_{2}}\right)
$$

by (21) and (33). For each $i, 0 \leqslant i \leqslant n-1$, we have that

$$
y \cdot x^{i}=-\varepsilon\left(\sum_{s=0}^{n-1}\left(\sum_{r+k=s} c_{k, 0} \beta_{r}\right) \cdot x^{i+s}\right)
$$

the terms for which $i+s \geqslant n$ drop out of this sum since $x^{n} \equiv-\varepsilon$ (expression in $x$ ), and $\varepsilon^{2}=0$. Among the remaining terms, we get a contribution to the trace whenever $x^{i}=x^{i+s}(\Rightarrow s=0)$, and this contribution is $-\varepsilon c_{0,0} \beta_{0}$. Since we get this contribution $n$ times $(0 \leqslant i \leqslant n-1)$, the trace is equal to $-n \in c_{(0,0)} \beta_{0}$, which has $\varepsilon$-coefficient

$$
d y_{[n]}\left(v_{2}\right)=-n c_{0,0} \beta_{0} \text {. }
$$

Adding these results, we see that

$$
\omega_{[n]}(v)=-\left(\sum_{r+k=n-1} b_{k, 0} \alpha_{r}\right)-n c_{0,0} \beta_{0} ;
$$

this expression is zero for all $v \in T H_{[Z]}$

$\Leftrightarrow$ the expression is zero for all choices of the $\alpha_{r}$ and $\beta_{0}$

$\Leftrightarrow$ the coefficients are all zero: $b_{k, 0}=0$ for $0 \leqslant k \leqslant n-1$ and $n \cdot c_{0,0}=0$, which proves the proposition.

To generalize (31) to surfaces $X$, it is not enough to consider the behavior of a symmetrized differential at a single point $[Z] \in H_{X}^{n}$ lying over $(n P) \in X(n)$; we must rather consider its behavior on the locus $\sigma_{n}^{-1}((n P))$ of all the points [Z] lying over $(n P)$. We recall the following (remarkable) fact concerning this locus:

(34) $\sigma_{n}^{-1}((n P))$ is irreducible (Briançon) and of dimension $n-1$ [7, Chapter 5, and Theorem 2.13].

As in Proposition 10, let $x, y$ be local parameters at $P \in X$, and $\omega=h_{1} d x+h_{2} d y$ be a holomorphic 1-form on $X$, where $h_{1}$ and $h_{2}$ have the power series expansions $\left(\Sigma=\Sigma_{0 \leqslant i, j}\right)$ :

$$
h_{1}=\sum b_{i, j} x^{i} y^{j}, \quad h_{2}=\sum c_{i, j} x^{i} y^{j}
$$


Let $I_{\alpha_{1}, \ldots, \alpha_{n-1}}=\left(x^{n}, y-\alpha_{1} \cdot x-\cdots-\alpha_{n-1} \cdot x^{n-1}\right)$, an ideal of colength $n$ in $O_{P}$ for each choice of the scalars $\alpha_{i}$, and let $Z_{\alpha_{1}, \ldots, \alpha_{n-1}}=\operatorname{Spec} O_{P} / I_{\alpha_{1}, \ldots, \alpha_{n-1}}$. The locus $G$ (for "generic type") of all the points $\left[Z_{\alpha_{1}, \ldots, \alpha_{n-1}}\right]$ has dimension $n-1$; consequently, (35) $G$ is an open dense subset of $\sigma_{n}^{-1}((n P))$.

We may now prove

THEOREM 7. The symmetrized differential $\omega_{[n]}$ is zero at every point of $\sigma_{n}^{-1}((n P)) \subset$ $H_{X}^{n} \Leftrightarrow$

$$
\begin{aligned}
b_{i, j}=c_{i, j} & =0 \quad \text { for } 0 \leqslant i+j \leqslant n-2, \\
b_{n-1,0} & =0 \\
b_{n-2,1}+c_{n-1,0} & =0 \\
b_{n-3,2}+c_{n-2,1} & =0 \\
\vdots & \\
b_{0, n-1}+c_{1, n-2} & =0 \\
c_{0, n-1} & =0 .
\end{aligned}
$$

Proof. By (35), it is enough to prove: $\omega_{[n]}$ is zero on $G \Leftrightarrow$ the conditions (36) hold. To do this, we first find the conditions imposed on the coefficients $b_{i, j}, c_{i, j}$ when $\omega_{[n]}$ is required to be zero at a particular point $\left[Z_{\alpha_{1}, \ldots, \alpha_{n-1}}\right] \in G$ (these are polynomial relations in the $b$ 's, $c$ 's, and $\alpha$ 's). We then show that requiring these relations to hold for all choices of the $\alpha$ 's is equivalent to imposing the conditions (36) on $\omega$.

For the first step, fix a point $\left[Z_{\alpha_{1}, \ldots, \alpha_{n-1}}\right] \in G$, and make the change of coordinates

$$
x=x, \quad y^{\prime}=y-\alpha_{1} x-\cdots-\alpha_{n-1} x^{n-1} .
$$

Rewriting $\omega$ in $x, y^{\prime}$ coordinates, we get

$$
\begin{aligned}
\omega & =h_{1} d x+h_{2} d y \\
& =h_{1} d x+h_{2}\left(d y^{\prime}+\left(\sum_{t=1}^{n-1} t \alpha_{t} x^{t-1}\right) d x\right) \\
& =\left(h_{1}+h_{2}\left(\sum_{t=1}^{n-1} t \alpha_{t} x^{t-1}\right)\right) d x+h_{2} d y^{\prime}
\end{aligned}
$$

expressing $h_{1}$ and $h_{2}$ as power series in $x$ and $y^{\prime}$, this becomes $\left(\Sigma=\Sigma_{0 \leqslant i, j}\right)$

$$
\begin{aligned}
\omega= & {\left[\sum b_{i, j} x^{i}\left(y^{\prime}+\sum_{t=1}^{n-1} \alpha_{t} x^{t}\right)^{j}\right.} \\
& \left.+\left(\sum c_{i, j} x^{i}\left(y^{\prime}+\sum_{t=1}^{n-1} \alpha_{t} x^{t}\right)^{j}\right) \cdot\left(\sum_{t=1}^{n-1} t \alpha_{t} x^{t-1}\right)\right] d x \\
& +\left(\sum c_{i, j} x^{i}\left(y^{\prime}+\sum_{t=1}^{n-1} \alpha_{t} x^{t}\right)^{j}\right) d y^{\prime}
\end{aligned}
$$


Let $\beta_{k}$ be the coefficient of $x^{k}$ in the power series multiplying $d x$ in (37). Since the ideal of $Z_{\alpha_{1}, \ldots, \alpha_{n-1}}$ in $x, y^{\prime}$ coordinates is $\left(x^{n}, y^{\prime}\right) \subset O_{P}$, Proposition 10 implies that

(38) $\omega_{[n]}$ is zero at $\left[Z_{\alpha_{1}, \ldots, \alpha_{n-1}}\right] \Leftrightarrow \beta_{0}=\beta_{1}=\cdots=\beta_{n-1}=0$ and $n \cdot c_{0,0}=0$; although not made explicit, these are the polynomial relations we had in mind.

We now show by induction on $k$ that requiring $\beta_{k}(0 \leqslant k \leqslant n-1)$ to be zero for all choices of $\alpha_{1}, \ldots, \alpha_{n-1}$ is equivalent to the conditions (36). First note that the "pure $x$ " part of the $d x$-coefficient in (37) is given by

$$
\begin{array}{r}
\sum_{0 \leqslant i, j} b_{i, j} x^{i}\left(\sum_{t=1}^{n-1} \alpha_{t} x^{t}\right)^{j}+\left(\sum_{0 \leqslant i, j} c_{i, j} x^{i}\left(\sum_{t=1}^{n-1} \alpha_{t} x^{t}\right)^{j}\right) \cdot\left(\sum_{t=1}^{n-1} t \alpha_{t} x^{t-1}\right) \\
=\sum_{0 \leqslant i, j} x^{i}\left(\sum_{t=1}^{n-1} \alpha_{t} x^{t}\right)^{j} \cdot\left(b_{i, j}+c_{i, j}\left(\sum_{t=1}^{n-1} t \alpha_{t} x^{t-1}\right)\right)
\end{array}
$$

after factoring. By the multinomial theorem, this becomes

$$
\begin{aligned}
\sum_{0 \leqslant i, j}\left(\sum_{s_{1}+\cdots+s_{n-1}=j}\left(\begin{array}{c}
j \\
s_{1}, \ldots, s_{n-1}
\end{array}\right) \alpha_{1}^{s_{1}} \cdots \alpha_{n-1}^{s_{n-1}} x^{\left(i+s_{1}+2 s_{2}+\cdots+(n-1) s_{n-1}\right)}\right) \\
\\
\cdot\left(b_{i, j}+c_{i, j}\left(\sum_{t=1}^{n-1} t \alpha_{t} x^{t-1}\right)\right) .
\end{aligned}
$$

For a given choice of $i$ and $j$ in the above sum, we call $i+j$ the weight of the associated terms. It is then clear that $\beta_{k}$, the coefficient of $x^{k}$ in (39), can only involve terms of weight $\leqslant k$. This, together with our induction hypotheses, will simplify the computations.

Step $k=0$. By inspection of (39), we see that

$$
\beta_{0}=b_{0,0}+\alpha_{1} \cdot c_{0,0}
$$

this equals zero for all choices of $\alpha_{1} \Leftrightarrow b_{0,0}=c_{0,0}=0$, which are the conditions of weight 0 in (36).

Induction Step. Suppose that we have shown that $\beta_{0}=\beta_{1}=\cdots=\beta_{k-1}=0$ $(0<k<n)$, for all choices of the $\alpha$ 's, is equivalent to the conditions

$$
\begin{aligned}
b_{i, j}=c_{i, j} & =0 \quad \text { for } 0 \leqslant i+j \leqslant k-2, \\
b_{k-1,0} & =0, \\
b_{k-2,1}+c_{k-1,0} & =0 \\
b_{k-3,2}+c_{k-2,1} & =0, \\
\vdots & \\
b_{0, k-1}+c_{1, k-2} & =0, \\
c_{0, k-1} & =0 .
\end{aligned}
$$

To complete the proof, we will show that if we assume $\beta_{0}=\beta_{1}=\cdots=\beta_{k-1}=0$ for all choices of the $\alpha$ 's $\left(\Leftrightarrow(40)\right.$ ), then assuming in addition that $\beta_{k}=0$ for all $\alpha$ 's is equivalent to the conditions having the same pattern as (40) with $k$ in place of 
$k-1$. Since $\beta_{k}$ depends only on the terms of weight $\leqslant k$, and since $b_{i, j}=c_{i, j}=0$ for $i+j \leqslant k-2$ by our assumption, only terms of weight $k$ or $k-1$ will appear in $\beta_{k}$. With this in mind, we read from (39) that

$$
\begin{aligned}
& \beta_{k}=\sum_{i+j=k}\left(\sum_{\substack{s_{1}+\cdots+s_{n-1}=j \\
i+s_{1}+2 s_{2}+\cdots+(n-1) s_{n-1}=k}}\left(\begin{array}{c}
j \\
s_{1}, \ldots, s_{n-1}
\end{array}\right) \alpha_{1}^{s_{1}} \cdots \alpha_{n-1}^{s_{n-1}}\left(b_{i, j}+\alpha_{1} c_{i, j}\right)\right) \\
& +\sum_{i+j=k-1}\left(\sum_{\substack{s_{1}+\cdots+s_{n-1}=j \\
i+s_{1}+2 s_{2}+\cdots+(n-1) s_{n-1}=k}}\left(\begin{array}{c}
j \\
s_{1}, \ldots, s_{n-1}
\end{array}\right) \alpha_{1}^{s_{1}} \cdots \alpha_{n-1}^{s_{n-1}}\left(b_{i, j}+\alpha_{1} c_{i, j}\right)\right) \\
& +\sum_{i+j=k-1}\left(\sum_{\substack{s_{1}+\cdots+s_{n-1}=j \\
i+s_{1}+2 s_{2}+\cdots+(n-1) s_{n-1}=k-1}}\left(\begin{array}{c}
j \\
s_{1}, \ldots, s_{n-1}
\end{array}\right) \alpha_{1}^{s_{1}} \cdots \alpha_{n-1}^{s_{n-1}}\left(2 \alpha_{2} c_{i, j}\right)\right) \text {. }
\end{aligned}
$$

By considering each summation in (41) separately (e.g., in the first sum, the conditions $i+j=k, s_{1}+\cdots+s_{n-1}=j$, and $i+s_{1}+2 s_{2}+\cdots+(n-1) s_{n-1}=$ $k \Rightarrow s_{1}=j, s_{2}=\cdots=s_{n-1}=0$ ), we see that the above sum equals

$$
\begin{aligned}
\sum_{i+j=k}\left(\alpha_{1}^{j} b_{i, j}+\alpha_{1}^{j+1} c_{i, j}\right) & +\sum_{\substack{i+j=k-1 \\
j \geq 1}}\left(j \alpha_{1}^{j-1} \alpha_{2} b_{i, j}+j \alpha_{1}^{j} \alpha_{2} c_{i, j}\right) \\
& +\sum_{i+j=k-1} 2 \alpha_{1}^{j} \alpha_{2} c_{i, j} .
\end{aligned}
$$

Regrouping this sum according to the various monomials in the $\alpha$ 's, we obtain

$$
\begin{aligned}
\beta_{k}= & b_{k, 0}+\sum_{j=1}^{k} \alpha_{1}^{j}\left(b_{k-j, j}+c_{k-j+1, j-1}\right)+\alpha_{1}^{k+1} \cdot c_{0, k} \\
& +\sum_{j=0}^{k-2}\left((j+1) b_{k-j-2, j+1}+(j+2) c_{k-j-1, j}\right) \cdot \alpha_{1}^{j} \cdot \alpha_{2} \\
& +(k+1) c_{0, k-1} \alpha_{1}^{k-1} \alpha_{2},
\end{aligned}
$$

where the last term is zero by (40). Since the distinct monomials in the $\alpha$ 's are algebraically independent, requiring $\beta_{k}$ to be zero for all choices of the $\alpha$ 's is equivalent to requiring that the coefficients of the monomials all vanish, i.e.,

$$
\begin{aligned}
b_{k, 0} & =0, \\
b_{k-1,1}+c_{k, 0} & =0, \\
b_{k-2,2}+c_{k-1,1} & =0, \\
\vdots & \\
b_{0, k}+c_{1, k-1} & =0, \\
c_{0, k} & =0,
\end{aligned}
$$


which are the conditions of weight $k$ we desired, and (at weight $k-1$ ),

$$
(j+1) b_{k-j-2, j+1}+(j+2) c_{k-j-1, j}=0
$$

for $0 \leqslant j \leqslant k-2$. But from (40) we see that

$$
b_{k-j-2, j+1}+c_{k-j-1, j}=0
$$

for $0 \leqslant j \leqslant k-2$, requiring these equations to hold simultaneously forces $b_{k-j-2, j+1}=c_{k-j-1, j}=0$ for $0 \leqslant j \leqslant k-2$, since the determinant

$$
\left|\begin{array}{cc}
j+1 & j+2 \\
1 & 1
\end{array}\right|=-1 \neq 0
$$

(in all characteristics). In view of (40), we now have that $b_{i, j}=c_{i, j}=0$ for $0 \leqslant i+j$ $\leqslant k-1$, as well as the desired conditions of weight $k$, so the induction step is complete and the theorem is proved.

REMARK. Since the expression (42) for $\beta_{k}$ only involves $\alpha_{1}$ and $\alpha_{2}$, we see $a$ posteriori that $\alpha_{3}, \ldots, \alpha_{n-1}$ are superfluous for the proof. Put another way, we have that $\omega_{[n]}$ is zero on all of $\sigma_{n}^{-1}((n P)) \Leftrightarrow \omega_{[n]}$ is zero at every point $\left[Z_{\alpha_{1}, \alpha_{2}, 0, \ldots, 0}\right] \Leftrightarrow$ the conditions (36) hold.

The generalization of (31) to surfaces which we have been seeking is given by the following

THEOREM 8. Let $X$ be an irreducible and nonsingular surface and let $\omega$ be $a$ holomorphic 1-form on $X$. If the $(n-1)$-jet of $\omega$ is zero at $P \in X$, then $\omega_{[n]}$ is zero at every point of $\sigma_{n}^{-1}((n P)) \subset H_{X}^{n}$. Moreover, if we assume in addition that $\omega$ is closed at $P$ and that $\operatorname{char}(k)=p \nmid n$, then the converse holds as well.

Proof. The first assertion follows immediately from Theorem 7:

the $(n-1)$-jet of $\omega$ is zero at $P \Rightarrow$ conditions (36) hold

$$
\Rightarrow \omega_{[n]} \text { is zero on } \sigma_{n}^{-1}((n P)) \text {. }
$$

(Alternative Proof. Suppose that the $(n-1)$ )-jet of $\omega=h_{1} d x+h_{2} d y$ is zero at $P \in X$. Then $h_{1}, h_{2} \in M_{P}^{n}$, and since $M_{P}^{n} \subset I$ for all ideals $I$ of colength $n$ in $O_{P}$ [7, Lemma 1.1], we have that $\hat{h}_{1}$ and $\hat{h}_{2}$ are each the zero map on $T H_{[Z]}$ for all $[Z] \in \sigma_{n}^{-1}((n P))$. The assertion now follows immediately from Corollary 2 of Theorem 1.)

We now prove the second assertion. In view of Theorem 7, we must prove that under the additional hypotheses given above, the conditions (36) imply the conditions $b_{i j}=c_{i j}=0$ for $0 \leqslant i+j \leqslant n-1$ (which say that the $(n-1)$-jet of $\omega$ at $P$ is zero). Since $\omega$ is closed $\left(\partial h_{1} / \partial y=\partial h_{2} / \partial x\right)$, the $b$ 's and $c$ 's of weight $n-1$ satisfy the relations

$$
(j+1) b_{n-j-2, j+1}-(n-j-1) c_{n-j-1, j}=0
$$

for $0 \leqslant j \leqslant n-2$; confronting these with the weight $n-1$ relations

$$
b_{n-j-2, j+1}+c_{n-j-1, j}=0
$$


for $0 \leqslant j \leqslant n-2$, from (36), we conclude that $b_{n-j-2, j+1}=c_{n-j-1, j}=0$ for $0 \leqslant j$ $\leqslant n-2$ (which proves what we want), since the determinant

$$
\left|\begin{array}{cc}
j+1 & -(n-j-1) \\
1 & 1
\end{array}\right|=n \neq 0
$$

(since we have assumed that $p \nmid n$ ).

6. Connection with generalized Weierstrass points. As noted in the Introduction, a Weierstrass point of a complete nonsingular curve $C$ is a point $P \in C$ for which the point $(g P)$ is a singularity of the Jacobian mapping $f_{g}: C(g) \rightarrow J$ (i.e. $g P$ is a special divisor), where $g$ is the genus of $C$. To bring this paper to a close, we show that (for one definition of generalized Weierstrass points) a similar interpretation is possible for the generalized Weierstrass points on a surface $X$, in terms of singularities of the Albanese mapping $\phi_{n}: H_{X}^{n} \rightarrow A$. Here we assume that $X$ is an irreducible and nonsingular projective surface/C. The following definition of the Weierstrass points on $X$ (which generalizes the classical definition for curves) is a special case of a general definition given in [9], which applies to varieties of any dimension and any bundles on those varieties. Let $J_{P}^{m}\left(\Omega_{X}^{1}\right)$ denote the $O_{P}$-module of $m$-jets of holomorphic 1-forms at $P \in X$.

Definition. $P \in X$ is a Weierstrass point of order $m$ (for the cotangent bundle $\left.\Omega_{X}^{1}\right) \Leftrightarrow$ the natural map

$$
j_{p}^{m}: \Gamma\left(\Omega_{X}^{1}\right) \rightarrow J_{p}^{m}\left(\Omega_{X}^{1}\right)
$$

(which takes a global 1 -form to its $m$-jet at $P$ ) is not of maximal rank.

In other words, $P$ is a Weierstrass point of order $m$ if more than the "expected number" of independent holomorphic 1 -forms on $X$ have zero $m$-jets at $P$. More precisely, if we let $K_{P}^{m} \subset \Gamma\left(\Omega_{X}^{1}\right)$ be the kernel of $j_{p}^{m}$, then

(43) $P \in X$ is a Weierstrass point of order $m \Leftrightarrow$

$$
\operatorname{dim}_{k}\left(K_{p}^{m}\right)>\max \{q-(m+1)(m+2), 0\},
$$

where $q=\operatorname{dim}_{k}\left(\Gamma\left(\Omega_{X}^{1}\right)\right)=\operatorname{dim} A$ is the irregularity of $X$, and (as is easily checked) $(m+1)(m+2)=\operatorname{dim}_{k}\left(J_{p}^{m}\left(\Omega_{X}^{1}\right)\right)$.

Since $X$ is projective/C, every global 1 -form on $X$ is closed, and (as previously noted) the Albanese mapping $f: X \rightarrow A$ induces by pullback an isomorphism $f^{*}$ : $\Gamma\left(\Omega_{A}^{1}\right) \rightarrow \Gamma\left(\Omega_{X}^{1}\right)[18, \S 9]$. Hence, by Theorem 8 ,

(44) $\omega \in K_{p}^{m} \Leftrightarrow \omega_{[m+1]}$ is zero at every point $[Z] \in \sigma_{m+1}^{-1}((m+1) P) \subset H_{X}^{m+1}$.

But, for nonzero $\omega$, the zeros of $\omega_{[m+1]}$ are singularities of $\varphi_{m+1}$, so (replacing $m+1$ by $n$ ) (44) suggests a theorem relating singularities of $\varphi_{n}: H_{X}^{n} \rightarrow A$ and Weierstrass points of order $n-1$ on $X$; it is now simply a matter of stating this theorem precisely.

To do this, let $0 \neq \omega \in \Gamma\left(\Omega_{X}^{1}\right)$, and let $\omega_{A} \in \Gamma\left(\Omega_{A}^{1}\right)$ be such that $\omega=f^{*}\left(\omega_{A}\right)$. Suppose that $\omega_{[n]}\left(=\varphi_{n}^{*}\left(\omega_{A}\right)\right.$, by (3)) is zero at every point $[Z] \in \sigma_{n}^{-1}((n P))$ for some $P \in X$, and let $a \in A$ be the image of $\sigma_{n}^{-1}((n P))$ under $\varphi_{n}$. Then, for each $[Z] \in$ $\varphi_{n}^{-1}((n P))$,

$$
D \varphi_{n}\left(T H_{[Z]}\right) \subset \operatorname{ker} \omega_{A} \subsetneq T A_{a},
$$


where by ker $\omega_{A}$ we mean the kernel of the covector induced by $\omega_{A}$ at $a$. Therefore,

$$
\left\langle\bigcup_{[Z] \in \sigma_{n}^{-1}((n P))} D \varphi_{n}\left(T H_{[Z]}\right)\right\rangle \subset \operatorname{ker} \omega_{A} \subsetneq T A_{a},
$$

where $\left\langle>\right.$ denotes linear span. Hence, the hypothesis that $\omega_{[n]}$ is zero everywhere on $\sigma_{n}^{-1}((n P))$ implies not merely that each $[Z] \in \sigma_{n}^{-1}((n P))$ is a singularity of $\varphi_{n}$, but something stronger-namely, that the images of all the tangent spaces $T H_{[Z]}$ lie in the same proper subspace of codimension 1 in $T A_{a}$. It is now easy to check that

(45) the codimension $\mu$ of $\left\langle\cup_{[Z] \in \sigma_{n}^{-1}}((n P)) D \varphi_{n}\left(T H_{[Z]}\right)\right\rangle$ in $T A_{a}$ is equal to the dimension of the subspace of all $\omega \in \Gamma\left(\Omega_{X}^{1}\right)$ for which $\omega_{[n]}$ is zero everywhere on $\sigma_{n}^{-1}((n P))$.

We can now state

THEOREM 9. Let $X$ be an irreducible and nonsingular projective surface of irregularity $q>0 / C$. Then $P \in X$ is a Weierstrass point of order $n-1 \Leftrightarrow$

$$
\mu>\max \{q-n(n+1), 0\},
$$

where $\mu$ is as in (45).

Proof. By (43), $P$ is Weierstrass of order $n-1 \Leftrightarrow$

$$
\operatorname{dim}\left(K_{p}^{n-1}\right)>\max \{q-n(n+1), 0\},
$$

but by (44) and (45), $\operatorname{dim}_{k}\left(K_{P}^{n-1}\right)=\mu$; the theorem follows.

EXAMPLE. Let $C$ be a complete nonsingular curve of genus 2 and let $A$ be its Jacobian. Let $P$ be a Weierstrass point on $C$, which means that a basis $\omega_{1}, \omega_{2}$ of $\Gamma\left(\Omega_{C}^{1}\right)$ may be chosen such that $\operatorname{ord}_{P}\left(\omega_{1}\right)=0, \operatorname{ord}_{P}\left(\omega_{2}\right)=2$. According to the definition above, $P$ is therefore a generalized Weierstrass point of order 1 on $C$, since $j_{P}^{1}: \Gamma\left(\Omega_{C}^{1}\right) \rightarrow J_{P}^{1}\left(\Omega_{C}^{1}\right)$ is not of maximal rank. ( $P$ is not a generalized Weierstrass point of any other order on $C$.)

Now consider the direct product $X=C \times C$, a surface of irregularity four. A basis of $\Gamma\left(\Omega_{X}^{1}\right)$ is obtained by pulling back the l-forms $\omega_{1}$ and $\omega_{2}$ by each of the two projection maps. Using this basis, one easily checks that $(P, P) \in C \times C$ is a generalized Weierstrass point of order 1 (and no other order) on $X$.

Finally, consider the symmetric product $X^{\prime}=C(2)$, a surface of irregularity two. As is well known, the Jacobian map $X^{\prime}=C(2) \rightarrow A$ represents $X^{\prime}$ as the blow-up of $A$ at a point $a \in A$; the exceptional locus $L$ represents the unique $g_{2}^{1}$ carried by $C$, which is the canonical system. The points $Q$ of $L$ (one of which is $(2 P)$ ) are generalized Weierstrass points of order 0 on $X^{\prime}$, which, since $\operatorname{dim} \Gamma\left(\Omega_{X^{\prime}}^{1}\right)=$ $\operatorname{dim} J_{Q}^{0}\left(\Omega_{X^{\prime}}^{1}\right)=2$, is a fancy way of saying that each point of $L$ is a zero of some global 1-form on $X^{\prime}$. (This last follows from (2), since $C(2) \rightarrow A$ is not smooth at any point of $L$. Theorem 9 is trivially verified in this case.) Using the basis of $\Gamma\left(\Omega_{X^{\prime}}^{1}\right)$ given by the symmetrizations of $\omega_{1}$ and $\omega_{2}$, and the analysis of symmetrized differentials given in [10], it can be shown that $(2 P)$ is not a generalized Weierstrass point of any higher order on $X^{\prime}$. Further analysis of the generalized Weierstrass points on $C(2)$ or $C(n)$ for curves of higher genus should be possible using [10], but that is beyond the scope of this (now concluded) paper. 


\section{REFERENCES}

1. A. Altman and S. Kleiman, Introduction to Grothendieck duality theory, Lecture Notes in Math., vol. 146, Springer-Verlag, Berlin, Heidelberg and New York, 1970.

2. A. Borel, Linear algebraic groups, Math. Lecture Notes Series, Benjamin, New York and Amsterdam, 1969.

3. J. Fogarty, Algebraic families on an algebraic surface, Amer. J. Math. 90 (1968), 511-521.

4. A. Grothendieck, Élements de géométrie algébrique, Inst. Hautes Études Sci. Publ. Math. 4 (1960); ibid 8 (1961); ibid 11 (1961); ibid 17 (1963); ibid 20 (1964); ibid 24 (1965); ibid 28 (1966); ibid 32 (1967).

5. , Fondaments de la geométrie algébrique, (Extraits du Sém. Bourbaki 1957-1962), Sécretariat Mathématique, Paris, 1962.

6. M. Huibregtse, The Albanese mapping for a punctual Hilbert scheme: I. Irreducibility of the fibers, Trans. Amer. Math. Soc. 251 (1979), 267-285.

7. A. Iarrobino, Punctual Hilbert schemes. Mem. Amer. Math. Soc. No. 10 (1977).

8. B. Iverson, Linear determinants with applications to the Picard scheme of a family of algebraic curves, Lecture Notes in Math., vol. 174, Springer-Verlag, Berlin and New York, 1970.

9. R. Ogawa, On the points of Weierstrass in dimensions greater than one, Trans. Amer. Math. Soc. 184 (1973), 401-417.

10. A. Mattuck and A. Mayer, The Riemann-Roch theorem for algebraic curves, Ann. Scuola Norm. Sup. Pisa (3) 17 (1963), 223-237.

11. D. Mumford, Rational equivalence of 0-cycles on surfaces, J. Math. Kyoto Univ. 92 (1969), 195-204.

12. ___ Abelian varieties, Oxford Univ. Press, Oxford, 1970.

13. Lectures on curves on an algebraic surface, Ann. of Math. Studies, no. 59, Princeton Univ. Press, Princeton, N.J., 1966.

14. A. A. Roitman, On $\Gamma$-equivalence of zero-dimensional cycles, Math. USSR-Sb. 15 (1971), 555-567.

15. I. R. Shafarevich, Basic algebraic geometry, Springer-Verlag, Berlin and New York, 1974.

16. J.-P. Serre, Groupes algébriques et corps de classes, Actualités Sci. Indust., no. 1264, Hermann, Paris, 1959.

17. __ Morphismes universels et variété d'Albanese, Séminaire C. Chevalley, E. N. S. 1958/59.

18. K. Ueno, Classification theory of algebraic varieties and compact complex spaces, Lecture Notes in Math., vol. 439, Springer-Verlag, Berlin and New York, 1975.

19. O. Zariski, Introduction to the problem of minimal models in the theory of algebraic surfaces, Publ. Math. Soc. Japan, Kenbryusha Printing Co., Ltd., Tokyo, 1958.

Department of Mathematics, Skidmore College, Saratoga Springs, New York 12866 